University of Wollongong

Research Online

Faculty of Informatics - Papers (Archive)

Faculty of Engineering and Information

Sciences

March 2004

\title{
Sharp results concerning the expression of functions as sums of finite differences
}

R. Nillsen

University of Wollongong, nillsen@uow.edu.au

S. Okada

University of Seville, Spain, okada@us.es

Follow this and additional works at: https://ro.uow.edu.au/infopapers

Part of the Physical Sciences and Mathematics Commons

\section{Recommended Citation}

Nillsen, R. and Okada, S.: Sharp results concerning the expression of functions as sums of finite differences 2004.

https://ro.uow.edu.au/infopapers/410

Research Online is the open access institutional repository for the University of Wollongong. For further information contact the UOW Library: research-pubs@uow.edu.au 


\title{
Sharp results concerning the expression of functions as sums of finite differences
}

\begin{abstract}
A square integrable function on the real line can be written as a finite sum of differences of order $s$ if and only if the Fourier transform of the function vanishes at the origin at a rate comparable with $|x|^{\wedge}\{-\mathrm{s}\}$. This enables us to see that the space of all such functions becomes a Hilbert space $D^{\wedge} s(R)$ and that this space is the range of the derivative of order $\mathrm{s}$ on the Sobolev space of order $\mathrm{s}$. It was proved by the author (Journal of Functional Analysis 110 (1992), 73-95) that each function in $D^{\wedge} s(R)$ is a sum of $2 s+1$ differences of order s. It was also known (Springer Lecture Notes in Mathematics vol. 1586, 1994) that in the case $s=1$, although every function in $D^{\wedge} 1(R)$ is a sum of 3 first order differences, there are functions in $D^{\wedge} 1(R)$ that cannot be written as the sum of 2 first order differences - that is, 3 is sharp as an estimate of the minimum number of differences required. It was an open problem as to whether in the case of $D^{\wedge} s(R)$, the number $2 s+1$ is sharp in this same sense. The main result in this paper shows that $2 s+1$ is sharp. That is, although every function in $D^{\wedge} s(R)$ is a sum of $2 s+1$ differences of order $s$, there are functions in $D^{\wedge} s(R)$ that cannot be written as the sum of $2 s$ differences of order $s$. In fact, substantially stronger results are proved, and results in related spaces of distributions are discussed. The main techniques involve the Fourier transform and combinatorial methods in harmonic analysis, in particular an estimate of the minimum potential of $n$ points scattered in the unit cube in $\mathrm{R}^{\wedge} \mathrm{s}$. This aspect generalizes work of Wolfgang Schmidt. Further ideas related to this work, the behaviour of the Fourier transform near the origin, and differential operators are on the author's website at http://www.uow.edu.au/ nillsen
\end{abstract}

\section{Keywords}

Fourier transform, Fourer series, finite differences, differences of order s, sharp estimate, Hilbert space, Banach space, Sobolev space, distributions

\section{Disciplines}

Physical Sciences and Mathematics

\section{Publication Details}

This article was originally published as: Nillsen, R \& Okada, S, Sharp results concerning the expression of functions as sums of finite differences, Proceedings of the London Mathematical Society, 2004, 88(2), 479-504. Copyright 2004 Cambridge University Press. Original journal can be found here. 


\title{
Sharp results concerning the expression of functions as sums of finite differences
}

\author{
Rodney Nillsen \\ School of Mathematics and Applied Statistics \\ University of Wollongong \\ Wollongong, NSW Australia 2522
}

\author{
Susumu Okada \\ School of Mathematics \\ University of New South Wales \\ Sydney, NSW Australia 2052*
}

\begin{abstract}
Let $s \in \mathbb{N}$, let $*$ denote convolution and let $\delta_{x}$ denote the Dirac measure at a point $x$. A function in $L^{2}(\mathbb{R})$ is called a difference of order $s$ if it is of the form $\sum_{k=0}^{s}(-1)^{k}\left(\begin{array}{l}s \\ k\end{array}\right) \delta_{k x} * g$ for some $x \in \mathbb{R}$ and $g \in L^{2}(\mathbb{R})$. In fact, the concept of a "difference of order $s$ " may be defined in a similar manner for each $s>0$. Then, it is known that a function $f$ in $L^{2}(\mathbb{R})$ is a finite sum of differences of order $s$ if and only if $\int_{\infty}^{\infty}|\widehat{f}(x)|^{2}|x|^{-2 s} d x<\infty$, and the vector space of all such functions is denoted by $\mathcal{D}_{s}\left(L^{2}(\mathbb{R})\right)$. Every function in $\mathcal{D}_{s}\left(L^{2}(\mathbb{R})\right)$ is a sum of $\operatorname{int}(2 s)+1$ differences of order $s$, where $\operatorname{int}(t)$ denotes the integer part of a real number $t$. Thus, every function in $\mathcal{D}_{1}\left(L^{2}(\mathbb{R})\right)$ is a sum of three first order differences, but it was proved in 1994 that there is a function in $\mathcal{D}_{1}\left(L^{2}(\mathbb{R})\right)$ which is never the sum of two first order differences. This complemented, for the group $\mathbb{R}$, the corresponding result for first order differences obtained by Meisters and Schmidt in 1972 for the circle group $\mathbb{T}$. In this paper, it is shown that there is a vector subspace of $L^{2}(\mathbb{R})$ which has dimension equal to that of the continuum such that, for each $s \geq 1 / 2$, every non-zero function in this subspace is a sum of int $(2 s)+1$ differences of order $s$ but is never the sum of int $(2 s)$ differences of order $s$. The proof depends upon extending to higher dimensions the following result set in two dimensions and obtained by Schmidt in 1972 in connection with Heilbronn's problem: if $x_{1}, \ldots, x_{n}$ are points in the unit square, $\sum_{1 \leq i<j \leq n}\left|x_{i}-x_{j}\right|^{-2} \geq 200^{-1} n^{2} \ln n$. Following on from the work of Meisters and Schmidt, the results presented here further develop a connection between certain estimates in combinatorial geometry and some questions of sharpness in harmonic analysis.
\end{abstract}

\section{Introduction}

Let $G$ be a locally compact abelian group, and let $L^{2}(G)$ denote the Hilbert space of all complex-valued, square integrable functions on $G$ with respect to the Haar measure on $G$.

\footnotetext{
*The authors wish to thank the Australian Research Council and the Mathematical Analysis Group at the University of Wollongong for generous suppport over the duration of this research.

AMS classification numbers: primary 42A38, secondary 52A40
} 
Let the Dirac measure at a point $x \in G$ be denoted by $\delta_{x}$, and let $*$ denote the operation of convolution where such an operation is defined. Thus, if $x \in G$ and $f \in L^{2}(G)$, then $\delta_{x} * f$ is given in $L^{2}(G)$ by

$$
\left(\delta_{x} * f\right)(t)=f(t-x),
$$

for almost all $t \in G$. A function on $G$ which is of the form $f-\delta_{x} * f$, for some $x \in G$ and $f \in L^{2}(G)$, is called a first order difference of $L^{2}(G)$. Then $\mathcal{D}_{1}\left(L^{2}(G)\right)$ denotes the vector subspace of $L^{2}(G)$ consisting of all finite sums of first order differences. In other words, a function $g$ in $L^{2}(G)$ belongs to $\mathcal{D}_{1}\left(L^{2}(G)\right)$ if and only if there exist an $n \in \mathbb{N}$, points $x_{1}, x_{2}, \ldots, x_{n} \in G$ and functions $f_{1}, f_{2}, \ldots, f_{n} \in L^{2}(G)$ such that

$$
g=\sum_{j=1}^{n}\left(f_{j}-\delta_{x_{j}} * f_{j}\right)
$$

Now, let the dual group of $G$ be denoted by $\widehat{G}$. Letting $\mathbb{R}$ denote the additive group of real numbers, we have $\widehat{\mathbb{R}}=\mathbb{R}$. Also, letting $\mathbb{T}$ denote the circle group consisting of all complex numbers with modulus one, we have $\widehat{\mathbb{T}}=\mathbb{Z}$, the group of all integers. If $f \in L^{2}(G)$, then $\widehat{f}$ denotes the Fourier transform of $f$, which is a function in $L^{2}(\widehat{G})$ [11, Theorem 1.6.1].

In 1972 , G. Meisters and W. Schmidt [6] characterised the space $\mathcal{D}_{1}\left(L^{2}(\mathbb{T})\right)$ as the subspace of $L^{2}(\mathbb{T})$ whose functions have Fourier transforms which have a certain behaviour at the identity 0 in the dual group $\mathbb{Z}$ of $\mathbb{T}$. More generally and specifically, they proved that if $G$ is a connected, compact abelian group and $\widehat{e}$ denotes the identity of $\widehat{G}$, then

$$
\mathcal{D}_{1}\left(L^{2}(G)\right)=\left\{f: f \in L^{2}(G) \text { and } \widehat{f}(\widehat{e})=0\right\} .
$$

The proof of (1.2) given in [6] revealed some fine detail concerning the representation of functions in the form (1.1). In particular, it was shown that for a compact connected abelian group $G$ the number $n$ in (1.1) may always be taken to be three. That is,

$$
\mathcal{D}_{1}\left(L^{2}(G)\right)=\left\{\sum_{j=1}^{3}\left(f_{j}-\delta_{x_{j}} * f_{j}\right): x_{1}, x_{2}, x_{3} \in G \text { and } f_{1}, f_{2}, f_{3} \in L^{2}(G)\right\} .
$$

However, for the circle group $\mathbb{T}$ the number three in (1.3) is sharp in the sense that it is shown in [6] that there is a function $f \in \mathcal{D}_{1}\left(L^{2}(\mathbb{T})\right)$ such that for all $x_{1}, x_{2} \in \mathbb{T}$ and all $f_{1}, f_{2} \in L^{2}(\mathbb{T})$

$$
f \neq \sum_{j=1}^{2}\left(f_{j}-\delta_{x_{j}} * f_{j}\right)
$$

A linear form $J$ on $L^{2}(G)$ is called translation invariant if $J\left(\delta_{x} * f\right)=J(f)$ for all $x \in G$ and $f \in L^{2}(G)$. Equation (1.2) shows that under the stated conditions, $\mathcal{D}_{1}\left(L^{2}(G)\right)$ has codimension one in $L^{2}(G)$. It then follows, as proved by Meisters and Schmidt, that if $G$ is connected, compact and abelian then every translation invariant linear form $J$ on $L^{2}(G)$ is a constant multiple of the Haar measure $G$, and so must be continuous. Therefore, (1.2) and (1.3) may be regarded as refinements of the statement that each translation invariant linear form on $L^{2}(G)$ is continuous. Further results in the compact group case also were obtained 
by Meisters [5]. Subsequently, B. Johnson [3] characterised those compact abelian groups $G$ for which every translation invariant linear form on $L^{2}(G)$ is continuous. In [2], J. Bourgain showed that every translation invariant linear form on $L^{p}(\mathbb{T}), 1<p<\infty$, is continuous, and the generalisation of this to certain compact abelian groups was studied by Lo [4].

Development of the preceding ideas for non-compact abelian groups has been carried out in $[7,8,9,10]$. However, the following remarks are restricted to the group $\mathbb{R}$. For each $s \in(0, \infty)$, a vector space $D_{s}\left(L^{2}(\mathbb{R})\right)$ may be defined to consist of all finite sums of functions which are "differences of order $s$ ". For $s=1$, a difference of order 1 is the same as a first order difference defined earlier. In the case $s=2$, a difference of order 2 , or a second order difference, is defined to be a function of the form $f-2^{-1}\left(\delta_{x}+\delta_{-x}\right) * f$ for some $x \in \mathbb{R}$ and $f \in L^{2}(\mathbb{R})$. Thus, a function $f$ in $L^{2}(\mathbb{R})$ belongs to $\mathcal{D}_{2}\left(L^{2}(\mathbb{R})\right)$ if and only if there are $n \in \mathbb{N}$, $x_{1}, x_{2}, \ldots, x_{n} \in \mathbb{R}$ and $f_{1}, f_{2}, \ldots, f_{n} \in L^{2}(\mathbb{R})$ such that

$$
f=\sum_{j=1}^{n}\left(f_{j}-2^{-1}\left(\delta_{x_{j}}+\delta_{x_{j}^{-1}}\right) * f_{j}\right) .
$$

For the spaces $\mathcal{D}_{s}\left(L^{2}(\mathbb{R})\right), s>0$, the result corresponding to (1.2) is the following:

$$
\mathcal{D}_{s}\left(L^{2}(\mathbb{R})\right)=\left\{f: f \in(\mathbb{R}) \text { and } \int_{-\infty}^{\infty} \frac{|\widehat{f}(x)|^{2}}{|x|^{2 s}} d x<\infty\right\} \text {. }
$$

It has been shown in [7, Theorem 2] and in [8, pp.80-81] (but see [9, Theorem 3] for a more accessible proof) that for each $s \in(0, \infty)$, every function $f$ in $\mathcal{D}_{s}\left(L^{2}(\mathbb{R})\right)$ is the sum of $\operatorname{int}(2 s)+1$ "differences of order $s$ " where int $(2 s)$ stands for the integer part of $2 s$. In particular, each function in $\mathcal{D}_{1}\left(L^{2}(\mathbb{R})\right)$ may be written as a sum of three first-order differences, and each function in $\mathcal{D}_{2}\left(L^{2}(\mathbb{R})\right)$ may be written as a sum of five second-order differences. These statements for the spaces $\mathcal{D}_{1}\left(L^{2}(\mathbb{R})\right)$ and $\mathcal{D}_{2}\left(L^{2}(\mathbb{R})\right)$ in the non-compact case of the group $\mathbb{R}$ correspond to the result in (1.3) which applies for the space $\mathcal{D}_{1}\left(L^{2}(\mathbb{T})\right)$ in the case of the compact group $\mathbb{T}$.

The question now arises as to whether or not the number int $(2 s)+1$ is sharp in estimating the number of "differences of order $s$ " needed to represent a general function in $\mathcal{D}_{s}\left(L^{2}(\mathbb{R})\right)$. In the case $s=1$, it was proved in [9, Proposition II.6.11] that 3 is a sharp estimate for the space $\mathcal{D}_{1}\left(L^{2}(\mathbb{R})\right)$ in the sense that there is a function $f$ in $\mathcal{D}_{1}\left(L^{2}(\mathbb{R})\right)$ such that for all $x_{1}, x_{2} \in \mathbb{R}$ and all $f_{1}, f_{2} \in L^{2}(\mathbb{R})$

$$
f \neq \sum_{j=1}^{2}\left(f_{j}-\delta_{x_{j}}+f_{j}\right) .
$$

So,(1.7) expresses the fact that the number 3 is sharp for $\mathcal{D}_{1}\left(L^{2}(\mathbb{R})\right)$, whereas (1.4) expresses the fact that the number 3 is sharp for $\mathcal{D}_{1}\left(L^{2}(\mathbb{T})\right)$. The main aim of this paper is to show that the number $\operatorname{int}(2 s)+1$ is a sharp estimate in each space $\mathcal{D}_{s}\left(L^{2}(\mathbb{R})\right), s \geq \frac{1}{2}$, in the following sense: every function in $\mathcal{D}_{s}\left(L^{2}(\mathbb{R})\right)$ is a sum of $\operatorname{int}(2 s)+1$ "differences of order $s$ ", but there is a function $f$ in $\mathcal{D}_{s}\left(L^{2}(\mathbb{R})\right)$ such that, for each $s \geq \frac{1}{2}, f$ is never the sum of $\operatorname{int}(2 s)$ "differences of order $s$ ". In particular, there is $f \in \mathcal{D}_{2}\left(L^{2}(\mathbb{R})\right)$ such that for all 
$x_{1}, x_{2}, x_{3}, x_{4} \in \mathbb{R}$ and $f_{1}, f_{2}, f_{3}, f_{4} \in L^{2}(\mathbb{R})$

$$
f \neq \sum_{j=1}^{4}\left(f_{j}-2^{-1}\left(\delta_{x_{j}}+\delta_{-x_{j}}\right)\right) * f_{j} .
$$

The proof of (1.4), to which (1.7) is a non-compact counterpart, was based by Meisters and Schmidt upon an earlier result of Schmidt [12] concerning the distribution of points in the unit square. The techniques used here for proving sharpness results such as (1.8) in the spaces $\mathcal{D}_{s}\left(L^{2}(\mathbb{R})\right), s \geq \frac{1}{2}$, depend upon extending this earlier result of Schmidt to $r$ dimensions. Letting $|\cdot|$ denote the usual Euclidean norm in $\mathbb{R}^{r}$, we prove the following result. When $r \in \mathbb{N}$, there is a constant $C_{r}>0$ such that for all $n \in \mathbb{N}$ and all distinct points $x_{1}, x_{2}, \ldots, x_{n} \in[0,1]^{r}$

$$
\sum_{1 \leq i<j \leq n} \frac{1}{\left|x_{i}-x_{j}\right|^{r}} \geq C_{r} n^{2} \log _{2} n .
$$

A connection is then made between this estimate and the divergence of certain integrals which determine whether a function can be expressed as the sum of a finite number of "differences of order $s$ ".

In fact, the preceding ideas are carried out in a more general way which constructs a vector space for which each non-zero function in the space is simultaneously in all of the spaces $\mathcal{D}_{s}\left(L^{2}(\mathbb{R})\right), s \geq 1 / 2$, but no such function can be expressed as a finite sum of $\operatorname{int}(2 s)$ "differences of order $s$ " for any $s \geq 1 / 2$. The ideas are then extended to spaces of distributions characterized by the requirement that their Fourier transforms are in some given space $L^{p}(\mathbb{R}), 1 \leq p<\infty$. In [13], T. Tao has described some applications of combinatorics in analysis, especially to the boundedness of Fourier integral operators. The methods of this paper further emphasise the rôle of combinatorial arguments in harmonic analysis.

\section{Differences of order $s$ and the spaces $\mathcal{D}_{s}\left(L^{2}(\mathbb{R})\right)$}

Let $\alpha: \mathbb{R} \rightarrow \mathbb{C}$ be a continuous, $2 \pi$-periodic function which has an absolutely convergent Fourier series. That is, if we let $\widehat{\alpha}(k)=(2 \pi)^{-1} \int_{-\pi}^{\pi} f(t) e^{-i k t} d t$ for all $k \in \mathbb{Z}$, then $\sum_{k=-\infty}^{\infty}|\widehat{\alpha}(k)|<\infty$. A function $f \in L^{2}(\mathbb{R})$ is called an $\alpha$-difference if there is $y \in \mathbb{R}$ and $g \in L^{2}(\mathbb{R})$ such that

$$
f=\sum_{-\infty}^{\infty} \widehat{\alpha}(k) \delta_{-k y} * g .
$$

Suppose, in addition, that there are numbers $s>0$ and $c_{1}, c_{2}>0$ such that

$$
c_{1}|x|^{s} \leq|\alpha(x)| \leq c_{2}|x|^{s}, \text { for all } x \in[-\pi, \pi] .
$$

Then an $\alpha$-difference will be called an $\alpha$-difference of order $s$. For example, let $\alpha_{1}(x)=$ $1-e^{-i x}$ for each $x \in \mathbb{R}$. Then, the function $\alpha_{1}$ is continuous and $2 \pi$-periodic, and it has an absolutely convergent Fourier series. Also, if $f \in L^{2}(\mathbb{R})$ and $x \in \mathbb{R}$,

$$
\sum_{-\infty}^{\infty} \widehat{\alpha}_{1}(k) \delta_{-k x} * f=f-\delta_{x} * f,
$$


so that an $\alpha_{1}$-difference is the same as a first order difference as defined in the Introduction. Also, since $\left|\alpha_{1}(x)\right|=2\left|\sin \frac{x}{2}\right|$, each $\alpha_{1}$-difference is an $\alpha_{1}$-difference of order 1 . So the first order differences are precisely the an $\alpha_{1}$-differences of order 1 , and our terminology is consistent.

A second example arises from considering the function $\alpha$ given by

$$
\alpha_{2}(x)=\left(1-e^{-i x}\right)^{2}=1-2 e^{-i x}+e^{-2 i x}, \text { for all } x \in \mathbb{R} .
$$

Letting $g=-2 \delta_{y} * f$, we have, for every $y \in \mathbb{R}$,

$$
\begin{aligned}
\sum_{k=-\infty}^{\infty} \widehat{\alpha}_{2}(k) \delta_{-k y} * f & =f-2 \delta_{y} * f+\delta_{2 y} * f \\
& =-\frac{1}{2} \delta_{-y} *\left(-2 \delta_{y} * f\right)+\left(-2 \delta_{y} * f\right)-\frac{1}{2} \delta_{y} *\left(-2 \delta_{y} * f\right) \\
& =g-\frac{1}{2}\left(\delta_{y} * g+\delta_{-y} * g\right) .
\end{aligned}
$$

This shows that the second order differences are the same as the $\alpha_{2}$-differences, and $\alpha_{2}$ differences are indeed $\alpha_{2}$-differences of order 2. The following theorem now relates general $\alpha$-differences to the behaviour of the Fourier transform along the lines described in the Introduction for first and second order differences. The most accessible proof of the theorem may be found in [9, Theorem 3] (alternatively, see [7, Theorem 2] or [8, Theorem II.4.6]).

Theorem 2.1 Let $s \in(0, \infty)$ and let $\alpha: \mathbb{R} \longrightarrow \mathbb{C}$ be a continuous $2 \pi$-periodic function which has an absolutely convergent Fourier series and is such that for some $c_{1}, c_{2}>0$, $c_{1}|x|^{s} \leq|\alpha(x)| \leq c_{2}|x|^{s}$ for all $x \in[-\pi, \pi]$. Let $\mathcal{D}_{\alpha}\left(L^{2}(\mathbb{R})\right)$ denote the vector space consisting of all functions in $L^{2}(\mathbb{R})$ which are finite sums of $\alpha$-differences. Then,

$$
\left\{\widehat{g}: g \in \mathcal{D}_{\alpha}\left(L^{2}(\mathbb{R})\right)\right\}=L^{2}\left(\mathbb{R},\left(1+|x|^{-2 s}\right) d x\right)
$$

Now (2.1) shows that the space $\mathcal{D}_{\alpha}\left(L^{2}(\mathbb{R})\right)$ in fact depends upon $s$ only, and not upon $\alpha$. Hence, when it exists, we denote the space $\mathcal{D}_{\alpha}\left(L^{2}(\mathbb{R})\right)$ by $\mathcal{D}_{s}\left(L^{2}(\mathbb{R})\right)$. However, for any $s>0$ there is a function $\alpha$ satisfying the assumptions of Theorem 2.1 , namely the $2 \pi$-periodic function on $\mathbb{R}$ which is given on $[-\pi, \pi]$ by $x \mapsto|\sin x / 2|^{s}$ (because this function has an absolutely convergent Fourier series, see [9, pp.71-73]). Alternatively, take the function on $[-\pi, \pi]$ given by $x \mapsto|x|^{s}$ and extended periodically to the whole of $\mathbb{R}$, which also has an absolutely convergent series $\left[8\right.$, p.71]. Thus, the space $\mathcal{D}_{s}\left(L^{2}(\mathbb{R})\right)$ is a vector subspace of $L^{2}(\mathbb{R})$ which is defined for all $s>0$. Note that for $x \in \mathbb{R}$ we denote the integer part of $x$ by $\operatorname{int}(x)$.

Theorem 2.2 Let $s \in(0, \infty)$. Then $\left\{\widehat{g}: g \in \mathcal{D}_{s}\left(L^{2}(\mathbb{R})\right)\right\}$ equals $L^{2}\left(\mathbb{R},\left(1+|x|^{-2 s}\right) d x\right)$, and $\mathcal{D}_{s}\left(L^{2}(\mathbb{R})\right)$ is a Hilbert space in the inner product $\langle\cdot, \cdot\rangle$ which is given by

$$
\langle f, g\rangle=\int_{-\infty}^{\infty} \widehat{f}(x) \overline{\widehat{g}(x)}\left(1+\frac{1}{|x|^{2 s}}\right) d x .
$$


Let $\alpha: \mathbb{R} \longrightarrow \mathbb{C}$ be a continuous $2 \pi$-periodic function which has an absolutely convergent Fourier series and for which there are $c_{1}, c_{2}>0$ such that $c_{1}|x|^{s} \leq|\alpha(x)| \leq c_{2}|x|^{s}$ for all $x \in[-\pi, \pi]$. Then, furthermore, every function in $\mathcal{D}_{s}\left(L^{2}(\mathbb{R})\right)$ is a sum of $\operatorname{int}(2 s)+1$ $\alpha$-differences.

The first part of Theorem 2.2 is immediate from Theorem 2.1. The most accessible proof of the remaining part is in [9, Theorem 3] (but see also [7, Theorem 2] and [8, Theorem II.4.4]).

In particular, it follows from Theorem 2.2 and (2.1) that any function in the space $\mathcal{D}_{1}\left(L^{2}(\mathbb{R})\right)$ is a sum of three first order differences. However, it is known [8, Proposition II.6.11] that there are functions in $\mathcal{D}_{1}\left(L^{2}(\mathbb{R})\right)$ which are never the sum of two first order differences. Also, from Theorem 2.2 and (2.2) it follows that any function in the space $\mathcal{D}_{2}\left(L^{2}(\mathbb{R})\right)$ is a sum of five second order differences. It is a purpose of this paper to show that there are functions in $\mathcal{D}_{2}\left(L^{2}(\mathbb{R})\right)$ which are never the sum of four first order differences. In general, it will be shown that when $s \geq 1 / 2$, the estimate of int $(2 s)+1$ as the required number of $\alpha$-differences in Theorem 2.2 is sharp - that is, there are functions in $\mathcal{D}_{s}\left(L^{2}(\mathbb{R})\right)$ which are never the sum of int $(2 s) \alpha$-differences, and in fact there are such functions which have this property simultaneously for all $s \geq 1 / 2$. Finally, note that a reason for interest in the spaces $\mathcal{D}_{s}\left(L^{2}(\mathbb{R})\right)$ is that they are the ranges of the appropriate powers of the differentiation operator on the corresponding Sobolev subspaces of $L^{2}(\mathbb{R})$. Specifically, the operator $(d / d x)^{s}$ maps the (possibly fractional) Sobolev subspace of order $s$ isometrically onto $\mathcal{D}_{s}\left(L^{2}(\mathbb{R})\right.$ ). The development of this aspect may be found for this and other operators in $[7,8,9,10]$.

\section{Points in the $r$-dimensional cube}

In [12], Schmidt proved that for any $n \geq 2$ and for any distinct points $x_{1}, x_{2}, \ldots, x_{n} \in[0,1]^{2}$

$$
\sum_{1 \leq i<j \leq n} \frac{1}{\left|x_{i}-x_{j}\right|^{2}} \geq \frac{1}{200} n^{2} \ln n .
$$

In this section, this result is extended so as to apply to $n$ points $x_{1}, x_{2}, \ldots, x_{n}$ in the $r$ dimensional cube $[0,1]^{r}$, where the exponent 2 in the term $\left|x_{i}-x_{j}\right|^{2}$ in (3.1) is replaced by an arbitrary exponent $2 s$ with $s>0$. The case where $r=2 s$ is a "borderline" one for estimating $\sum_{1 \leq i<j \leq n}\left|x_{i}-x_{j}\right|^{-2 s}$, and is the one of main interest.

Lemma 3.1 Let $q \in \mathbb{N}$ and let $c_{1}, d_{1}, c_{2}, d_{2}, \ldots, c_{q}, d_{q}$ be complex numbers. Then

$$
\sum_{k=1}^{q} c_{k} d_{k}=\left[\sum_{k=1}^{q-1}\left(c_{k+1}-c_{k}\right)\left(d_{k+1}+d_{k+2}+\cdots+d_{q}\right)\right]+c_{1}\left(d_{1}+d_{2}+\cdots+d_{q}\right) .
$$

(Here, if $q=1$, the term in the square bracket is taken to be 0 .)

Proof. The statement is easily checked for $q=1$ and $q=2$. So it may be proved by induction, or simply by rearranging the terms on the right hand side of the equation. 
Letting $r \in \mathbb{N}$, the usual Euclidean distance between $x, y \in \mathbb{R}^{r}$ is denoted by $|x-y|$. If $A$ is a finite set, then let $|A|$ denote the number of elements in $A$. If $x_{1}, x_{2}, \ldots, x_{n}$ are $n$ points in $\mathbb{R}^{r}$ and if $c>0$, let

$$
\mu\left(c ; x_{1}, x_{2}, \ldots, x_{n}\right)=\mid\left\{(i, j): 1 \leq i<j \leq n \text { and }\left|x_{i}-x_{j}\right| \leq c\right\} \mid .
$$

It is useful to note that if $x_{1}, x_{2}, \ldots x_{n}$ are $n$ distinct points in $[0,1]^{r}$ and if $c$ is strictly between 0 and $\min \left\{\left|x_{i}-x_{j}\right|: 1 \leq i<j \leq n\right\}$, then $\mu\left(c ; x_{1}, x_{2}, \ldots, x_{n}\right)=0$. So, it follows that when $x_{1}, x_{2}, \ldots, x_{n}$ are distinct, we have $\mu\left(c ; x_{1}, x_{2}, \ldots, x_{n}\right)=0$ for all sufficiently small $c>0$.

Lemma 3.2 Let $s>0$ and let $r, n \in \mathbb{N}$ with $n \geq 2$. Suppose that $x_{1}, x_{2}, \ldots, x_{n}$ are $n$ distinct points in $[0,1]^{r}$. Then

$$
\sum_{1 \leq i<j \leq n} \frac{1}{\left|x_{i}-x_{j}\right|^{2 s}} \geq \frac{2^{2 s}-1}{r^{s}}\left(\sum_{k=1}^{\infty} 2^{2 s(k-1)} \mu\left(\sqrt{r} 2^{-k} ; x_{1}, x_{2}, \ldots, x_{n}\right)\right) .
$$

Proof. As $x_{1}, x_{2}, \ldots, x_{n}$ are distinct and as $n \geq 2$, we may define

$$
q=\min \left\{k \in \mathbb{N}: \sqrt{r} 2^{-k}<\left|x_{i}-x_{j}\right| \text { for all } 1 \leq i<j \leq n\right\} .
$$

Apart from the fact that $q \geq 1$, the number $q$ satisfies

$$
\sqrt{r} 2^{-q}<\left|x_{i}-x_{j}\right| \leq \sqrt{r}
$$

for all $1 \leq i<j \leq n$. Now, observe that

$$
\left(\sqrt{r} 2^{-q}, \sqrt{r}\right]=\bigcup_{k=1}^{q}\left(\sqrt{r} 2^{-k}, \sqrt{r} 2^{-(k-1)}\right]
$$

where the union on the right hand side is disjoint. Make the definition that for each $k=$ $1,2, \ldots, q$,

$$
A_{k}=\left\{(i, j): 1 \leq i<j \leq n \text { and } \sqrt{r} 2^{-k}<\left|x_{i}-x_{j}\right| \leq \sqrt{r} 2^{-(k-1)}\right\} .
$$

It follows from (3.4), (3.5) and (3.6) that

$$
\bigcup_{k=1}^{q} A_{k}=\{(i, j): 1 \leq i<j \leq n\}
$$

and that for each $q \geq 2$ and $\ell=0,1, \ldots, q-1$ we have

$$
\bigcup_{k=\ell+1}^{q} A_{k}=\left\{(i, j): 1 \leq i<j \leq n \text { and }\left|x_{i}-x_{j}\right| \leq \sqrt{r} 2^{-\ell}\right\}
$$


Hence, for $\ell=0,1, \ldots, q-1$,

$$
\mu\left(\sqrt{r} 2^{-\ell} ; x_{1}, x_{2}, \ldots, x_{n}\right)=\left|\bigcup_{k=\ell+1}^{q} A_{k}\right| .
$$

Now, Lemma 3.1, (3.7) and (3.8) give

$$
\begin{aligned}
\sum_{1 \leq i<j \leq n} \frac{1}{\left|x_{i}-x_{j}\right|^{2 s}} & =\sum_{k=1}^{q}\left(\sum_{(i, j) \in A_{k}} \frac{1}{\left|x_{i}-x_{j}\right|^{2 s}}\right) \\
& \geq \sum_{k=1}^{q}\left(\sum_{(i, j) \in A_{k}} \frac{2^{2 s(k-1)}}{r^{s}}\right) \\
& =\frac{1}{r^{s}} \sum_{k=1}^{q} 2^{2 s(k-1)}\left|A_{k}\right| \\
& \geq \frac{1}{r^{s}}\left(\sum_{k=1}^{q-1}\left(2^{2 s k}-2^{2 s(k-1)}\right)\left(\left|A_{k+1}\right|+\ldots+\left|A_{q}\right|\right)\right) \\
& =\frac{2^{2 s}-1}{r^{s}} \sum_{k=1}^{q-1} 2^{2 s(k-1)} \mu\left(\sqrt{r} 2^{-k} ; x_{1}, x_{2}, \ldots, x_{n}\right) \\
& =\frac{2^{2 s}-1}{r^{s}} \sum_{k=1}^{\infty} 2^{2 s(k-1)} \mu\left(\sqrt{r} 2^{-k} ; x_{1}, x_{2}, \ldots, x_{n}\right),
\end{aligned}
$$

where the last step follows from the definition of $q$ in (3.3).

Let $m, r \in \mathbb{N}$. Then the cube $[0,1]^{r}$ may be expressed as a disjoint union of $m^{r}$ cubes each having sides of length $1 / m$ and volume $m^{-r}$. For each $m \in \mathbb{N}$ let $\mathcal{C}_{m}$ be one such family of cubes. Given $n \in \mathbb{N}$, if $x_{1}, x_{2}, \ldots, x_{n}$ are $n$ points in $[0,1]^{r}$ and if $\rho \in \mathcal{C}_{m}$, then make the definition that

$$
\nu\left(\rho ; x_{1}, x_{2}, \ldots, x_{n}\right)=\mid\left\{i: 1 \leq i \leq n \text { and } x_{i} \in \rho\right\} \mid .
$$

Lemma 3.3 Let $0<c<1$, and let $r, m, n \in \mathbb{N}$ with $n \geq c^{-1} m^{r}$. Let $[0,1]^{r}$ be expressed as a disjoint union of cubes in a given family $\mathcal{C}_{m}$, as described above. Then the following statements hold for any given $n$ points $x_{1}, x_{2}, \ldots, x_{n}$ in $[0,1]^{r}$.

$$
\begin{aligned}
& \text { (i) } \sum_{\rho \in \mathcal{C}_{m}} \nu\left(\rho ; x_{1}, x_{2}, \ldots, x_{n}\right) \cdot\left(\nu\left(\rho ; x_{1}, x_{2}, \ldots, x_{n}\right)-1\right) \geq \frac{(1-c)^{2} n^{2}}{m^{r}} . \\
& \text { (ii) } \mu\left(\frac{\sqrt{r}}{m} ; x_{1}, x_{2}, \ldots, x_{n}\right) \geq \frac{(1-c)^{2} n^{2}}{2 m^{r}} .
\end{aligned}
$$

Proof. (i) Let

$$
\mathcal{C}_{m}^{\prime}=\left\{\rho: \rho \in \mathcal{C}_{m} \text { and } \nu\left(\rho ; x_{1}, x_{2}, \ldots, x_{n}\right)>0\right\}
$$


Put $\ell=\left|\mathcal{C}_{m}^{\prime}\right| \in \mathbb{N}$. Then, an application of the Cauchy-Schwartz inequality in $\mathbb{R}^{\ell}$ gives

$$
\begin{aligned}
& \sum_{\rho \in \mathcal{C}_{m}} \nu\left(\rho ; x_{1}, x_{2}, \ldots, x_{n}\right) \cdot\left(\nu\left(\rho ; x_{1}, x_{2}, \ldots, x_{n}\right)-1\right) \\
= & \sum_{\rho \in \mathcal{C}_{m}^{\prime}} \nu\left(\rho ; x_{1}, x_{2}, \ldots, x_{n}\right) \cdot\left(\nu\left(\rho ; x_{1}, x_{2}, \ldots, x_{n}\right)-1\right) \\
\geq & \sum_{\rho \in \mathcal{C}_{m}^{\prime}}\left(\nu\left(\rho ; x_{1}, x_{2}, \ldots, x_{n}\right)-1\right)^{2} \\
= & \frac{1}{\ell}\left(\sum_{\rho \in \mathcal{C}_{m}^{\prime}} 1\right)\left(\sum_{\rho \in \mathcal{C}_{m}^{\prime}}\left(\nu\left(\rho ; x_{1}, x_{2}, \ldots, x_{n}\right)-1\right)^{2}\right) \\
\geq & \frac{1}{\ell}\left(\sum_{\rho \in \mathcal{C}_{m}^{\prime}}\left(\nu\left(\rho ; x_{1}, x_{2}, \ldots, x_{n}\right)-1\right)\right)^{2} \\
= & \frac{1}{\ell}\left(\sum_{\rho \in \mathcal{C}_{m}^{\prime}} \nu\left(\rho ; x_{1}, x_{2}, \ldots, x_{n}\right)-\sum_{\rho \in \mathcal{C}_{m}^{\prime}} 1\right)^{2} \\
= & \frac{1}{\ell}\left(\sum_{\rho \in \mathcal{C}_{m}^{\prime}} \nu\left(\rho ; x_{1}, x_{2}, \ldots, x_{n}\right)-\ell\right)^{2} \\
= & \frac{1}{\ell}(n-\ell)^{2} \\
\geq & \frac{1}{m^{r}}\left(n-m^{r}\right)^{2} \\
\geq & \frac{1}{m^{r}}(n-n c)^{2} \\
= & \frac{(1-c)^{2} n^{2}}{m^{r}} . \\
a &
\end{aligned}
$$

(ii) Let $\rho \in \mathcal{C}_{m}$, and observe that if $i, j \in\{1,2, \ldots n\}$ we have

$$
x_{i}, x_{j} \in \rho \Longrightarrow\left|x_{i}-x_{j}\right| \leq \frac{\sqrt{r}}{m} .
$$

Thus, if $1 \leq i<j \leq n$ and $x_{i}, x_{j} \in \rho$, we have

$$
(i, j) \in\left\{(k, l): 1 \leq k<l \leq n \text { and }\left|x_{k}-x_{l}\right| \leq \frac{\sqrt{r}}{m}\right\}
$$

Now, there are $2^{-1} \nu\left(\rho ; x_{1}, x_{2}, \ldots, x_{n}\right)\left(\nu\left(\rho ; x_{1}, x_{2}, \ldots, x_{n}\right)-1\right)$ points $(i, j)$ with $1 \leq i<j \leq n$ and $x_{i}, x_{j} \in \rho$. It therefore follows from (3.9) and the result (i), already proved, that

$\mu\left(\frac{\sqrt{r}}{m} ; x_{1}, x_{2}, \ldots x_{n}\right) \geq \sum_{\rho \in \mathcal{C}_{m}} 2^{-1} \nu\left(\rho ; x_{1}, x_{2}, \ldots, x_{n}\right)\left(\nu\left(\rho ; x_{1}, x_{2}, \ldots, x_{n}\right)-1\right) \geq \frac{(1-c)^{2} n^{2}}{2 m^{r}}$ 
Theorem 3.4 Let $r \in \mathbb{N}$. Then the following statements hold.

(i) If $s \in(0, \infty)$, if $n \geq 2$ and $x_{1}, x_{2}, \ldots, x_{n}$ are distinct elements in $[0,1]^{r}$, then

$$
\sum_{1 \leq i<j \leq n} \frac{1}{\left|x_{i}-x_{j}\right|^{2 s}} \geq \frac{n^{2}}{4 r^{s}} \text {. }
$$

(ii) There is $C_{r}>0$ such that for all $n \geq 2$ and all distinct elements $x_{1}, x_{2}, \ldots, x_{n}$ of $[0,1]^{r}$,

$$
\sum_{1 \leq i<j \leq n} \frac{1}{\left|x_{i}-x_{j}\right|^{r}} \geq C_{r} \cdot n^{2} \log _{2} n .
$$

(iii) If $s \in(0, \infty)$ and $r<2 s$, there is $D_{r, s}>0$ such that for all $n \geq 2$ and all distinct elements $x_{1}, x_{2}, \ldots, x_{n}$ of $[0,1]^{r}$,

$$
\sum_{1 \leq i<j \leq n} \frac{1}{\left|x_{i}-x_{j}\right|^{2 s}} \geq D_{r, s} \cdot n^{1+2 s / r} .
$$

Proof. (i) If $x_{i}, x_{j}$ are distinct elements of $[0,1]^{r}$, then $\left|x_{i}-x_{j}\right| \leq \sqrt{r}$. So, if $x_{1}, x_{2}, \ldots, x_{n}$ are distinct elements in $[0,1]^{r}$,

$$
\sum_{1 \leq i<j \leq n} \frac{1}{\left|x_{i}-x_{j}\right|^{2 s}} \geq \sum_{1 \leq i<j \leq n} \frac{1}{r^{s}}=\frac{1}{r^{s}} \cdot \frac{n(n-1)}{2} \geq \frac{1}{r^{s}} \cdot \frac{n^{2}}{4} .
$$

(ii) and (iii) Let $0<c<1$ and let $k \in \mathbb{N}$. Applying Lemma 3.3 with $m=2^{k}$ gives:

$$
n \geq c^{-1} 2^{r k} \Longrightarrow \mu\left(\frac{\sqrt{r}}{2^{k}} ; x_{1}, x_{2}, \ldots, x_{n}\right) \geq \frac{(1-c)^{2}}{2^{r k+1}} n^{2} .
$$

That is,

$$
\frac{\log _{2} n c}{r} \geq k \Longrightarrow \mu\left(\frac{\sqrt{r}}{2^{k}} ; x_{1}, x_{2}, \ldots, x_{n}\right) \geq \frac{(1-c)^{2}}{2^{r k+1}} n^{2} .
$$

Now, apply Lemma 3.2 together with (3.10). Then it follows that if $n \geq 2^{r} / c$,

$$
\begin{aligned}
\sum_{1 \leq i<j \leq n} \frac{1}{\left|x_{i}-x_{j}\right|^{2 s}} & \geq \frac{2^{2 s}-1}{r^{s}}\left(\sum_{1 \leq k \leq\left(\log _{2} n c\right) / r} 2^{2 s(k-1)}(1-c)^{2} n^{2} 2^{-r k-1}\right) \\
& =\frac{2^{2 s}-1}{2^{r+1} r^{s}} \cdot(1-c)^{2} n^{2}\left(\sum_{k=1}^{\operatorname{int}\left(\left(\log _{2} n c\right) / r\right)} 2^{(2 s-r)(k-1)}\right) .
\end{aligned}
$$

We consider now when $2 s=r \in \mathbb{N}$. In this case (3.11) gives the fact that if $n \geq 2^{r} / c$, then

$$
\begin{aligned}
\sum_{1 \leq i<j \leq n} \frac{1}{\left|x_{i}-x_{j}\right|^{r}} & \geq \frac{2^{r}-1}{2^{r+1} r^{r / 2}} \cdot(1-c)^{2} n^{2} \cdot \operatorname{int}\left(\frac{\log _{2} n c}{r}\right) \\
& \geq \frac{2^{r}-1}{2^{r+1} r^{r / 2}} \cdot(1-c)^{2} n^{2}\left(\frac{\log _{2} n c}{r}-1\right) \\
& =\frac{2^{r}-1}{2^{r+1} r^{r / 2}} \cdot(1-c)^{2} n^{2} \frac{1}{r}\left(\log _{2} n c-\log _{2} 2^{r}\right) \\
& =\frac{2^{r}-1}{2^{r+1} r^{1+r / 2}} \cdot(1-c)^{2} n^{2} \log _{2}\left(\frac{n c}{2^{r}}\right) .
\end{aligned}
$$


Let $\theta$ be any given number in $(0,1)$. We have

$$
n>\left(\frac{2^{r}}{c}\right)^{1 /(1-\theta)} \Longrightarrow \log _{2}\left(\frac{n c}{2^{r}}\right)>\theta \log _{2} n .
$$

It follows from (3.12) and (3.13) that if $n>\left(2^{r} / c\right)^{1 /(1-\theta)}$, then

$$
\sum_{1 \leq i<j \leq n} \frac{1}{\left|x_{i}-x_{j}\right|^{r}} \geq \frac{\left(2^{r}-1\right)(1-c)^{2} \theta}{2^{r+1} r^{1+r / 2}} \cdot n^{2} \log _{2} n .
$$

Observe also that for $2 \leq n \leq\left(2^{r} / c\right)^{1 /(1-\theta)}$,

$$
n^{2} \geq \frac{n^{2} \log _{2} n}{\log _{2}\left(2^{r} / c\right)^{1 / 1-\theta}}=\frac{1-\theta}{r-\log _{2} c} \cdot n^{2} \log _{2} n .
$$

So, by part (i), which has already been proved, we have in this case that

$$
\sum_{1 \leq i<j \leq n} \frac{1}{\left|x_{i}-x_{j}\right|^{r}} \geq \frac{1}{4 r^{r / 2}} \cdot \frac{1-\theta}{r-\log _{2} c} \cdot n^{2} \log _{2} n
$$

It is now immediate from (3.14) and (3.15) that for all $n \geq 2$,

$$
\sum_{1 \leq i<j \leq n} \frac{1}{\left|x_{i}-x_{j}\right|^{r}} \geq \frac{1}{4 r^{r / 2}} \min \left(\frac{1-\theta}{r-\log _{2} c}, \frac{2 \theta(1-c)^{2}\left(1-2^{-r}\right)}{r}\right) \cdot n^{2} \log _{2} n .
$$

So, if

$$
C_{r}=\frac{1}{4 r^{r / 2}} \min \left(\frac{1-\theta}{r-\log _{2} c}, \frac{2 \theta(1-c)^{2}\left(1-2^{-r}\right)}{r}\right),
$$

we have that for all $n \geq 2$ and all distinct $x_{1}, x_{2}, \ldots, x_{n} \in \mathbb{R}^{r}$,

$$
\sum_{1 \leq i<j \leq n} \frac{1}{\left|x_{i}-x_{j}\right|^{r}} \geq C_{r} n^{2} \log _{2} n .
$$

Thus, (ii) has been proved.

Now, to deduce (iii), assume that $r<2 s$. In this case we have from (3.11) that if $n \geq 2^{r} / c$, then

$$
\begin{aligned}
\sum_{1 \leq i<j \leq n} \frac{1}{\left|x_{i}-x_{j}\right|^{2 s}} & \geq \frac{2^{2 s}-1}{2^{r+1} r^{s}\left(2^{2 s-r}-1\right)} \cdot(1-c)^{2} \cdot n^{2}\left(2^{(2 s-r) \operatorname{int}\left(\left(\log _{2} n c\right) / r\right)}-1\right) \\
& \geq \frac{2^{2 s}-1}{2^{r+1} r^{s}\left(2^{2 s-r}-1\right)} \cdot(1-c)^{2} \cdot n^{2}\left(2^{(2 s-r)\left(\left(\log _{2} n c\right) / r-1\right)}-1\right) \\
& =\frac{2^{2 s}-1}{2^{r+1} r^{s}\left(2^{2 s-r}-1\right)} \cdot(1-c)^{2} \cdot n^{2}\left(2^{(2 s-r)\left(\left(\log _{2} n c / 2^{r}\right) / r\right)}-1\right) \\
& =\frac{2^{2 s}-1}{2^{r+1} r^{s}\left(2^{2 s-r}-1\right)} \cdot(1-c)^{2} \cdot n^{2}\left(2^{(2 s / r-1) \log _{2} n c / 2^{r}}-1\right)
\end{aligned}
$$




$$
\begin{aligned}
& =\frac{2^{2 s}-1}{2^{r+1} r^{s}\left(2^{2 s-r}-1\right)} \cdot(1-c)^{2} \cdot n^{2}\left(\left(\frac{n c}{2^{r}}\right)^{2 s / r-1}-1\right) \\
& =\frac{2^{2 s}-1}{2^{2 s+1} r^{s}\left(2^{2 s-r}-1\right)} \cdot(1-c)^{2} c^{2 s / r-1} \cdot n^{1+2 s / r}\left(1-\left(\frac{2^{r}}{n c}\right)^{2 s / r-1}\right) .
\end{aligned}
$$

Now, as in the proof of (ii), let $\theta \in(0,1)$ be given. We have

$$
n>\frac{2^{r}}{c} \cdot \frac{1}{(1-\theta)^{1 /(2 s / r-1)}} \Longrightarrow\left(\frac{2^{r}}{n c}\right)^{2 s / r-1}<1-\theta .
$$

It follows from (3.17) and (3.18) that if $n>2^{r} c^{-1}(1-\theta)^{-1 /(2 s / r-1)}$,

$$
\sum_{1 \leq i<j \leq n} \frac{1}{\left|x_{i}-x_{j}\right|^{2 s}} \geq \frac{2^{2 s}-1}{2^{2 s+1} r^{s}\left(2^{2 s-r}-1\right)} \cdot(1-c)^{2} c^{2 s / r-1} \theta \cdot n^{1+2 s / r} .
$$

On the other hand observe that for $2 \leq n \leq 2^{r} c^{-1}(1-\theta)^{-1 /(2 s / r-1)}$,

$$
n^{2} \geq(1-\theta) 2^{r-2 s} c^{2 s / r-1} n^{1+2 s / r} .
$$

So, by part (i), (3.19) and (3.20), we have that for all $n \geq 2$,

$$
\sum_{1 \leq i<j \leq n} \frac{1}{\left|x_{i}-x_{j}\right|^{2 s}} \geq \frac{1}{4 r^{s}} \min \left(\frac{1-\theta}{2^{2 s-r}} \cdot c^{2 s / r-1}, \frac{2^{2 s}-1}{2^{2 s-1}\left(2^{2 s-r}-1\right)} \cdot(1-c)^{2} c^{2 s / r-1} \theta\right) \cdot n^{1+2 s / r} .
$$

Thus, if we put

$$
D_{r, s}=\frac{c^{2 s / r-1}}{2^{2 s-r+2} r^{s}} \min \left(1-\theta, \frac{2^{2 s}-1}{2^{r-1}\left(2^{2 s-r}-1\right)} \cdot(1-c)^{2} \theta\right)
$$

we have that for all $n \geq 2$ and all distinct $x_{1}, x_{2}, \ldots, x_{n} \in \mathbb{R}^{n}$,

$$
\sum_{1 \leq i<j \leq n} \frac{1}{\left|x_{i}-x_{j}\right|^{2 s}} \geq D_{r, s} n^{1+2 s / r} .
$$

Thus, (iii) has been proved.

Note that in (i) of Theorem 3.4, the inequality is sharp in the sense that if $D$ is any number with $D>1 / 4 r^{s}$, then there are $n \in \mathbb{N}$ and $x_{1}, x_{2}, \ldots, x_{n} \in[0,1]^{r}$ such that $\sum_{1 \leq i<j \leq n}\left|x_{i}-x_{j}\right|^{-2 s}<D n^{2}$. To see this, simply take $n=2, x_{1}=(0,0, \ldots, 0)$ and $x_{2}=$ $(1,1, \ldots, 1)$ in $[0,1]^{r}$, and observe that $\left|x_{1}-x_{2}\right|^{-2 s}=2^{2} / 4 r^{s}$. Concerning the inequalities in (ii) and (iii) of Theorem 3.4, the situation is less clear. The constants $C_{r}$ and $D_{r, s}$ are given by (3.16) and (3.21) respectively, in which the numbers $c, \theta$ are in $(0,1)$ but are otherwise arbitrary. However, if $c$ is given in $(0,1)$, there is a unique value of $\theta$ in $(0,1)$ which maximises $C_{r}$ as given in (3.16), and similarly there is a unique $\theta$ in $(0,1)$ which maximises $D_{r, s}$ as given in (3.21). In the case of $C_{r}$ this maximum value becomes

$$
C_{r}=\frac{1-2^{-r}}{2 r^{r / 2}} \cdot \frac{(1-c)^{2}}{r+2\left(1-2^{-r}\right)(1-c)^{2}\left(r-\log _{2} c\right)} .
$$


If we take $c=1 / 4$, we obtain

$$
C_{r}=\frac{1}{4 r^{r / 2}} \cdot \frac{9\left(1-2^{-r}\right)}{8 r+9(r+2)\left(1-2^{-r}\right)} .
$$

Thus, if $r, n \in \mathbb{N}$ with $n \geq 2$, and if $x_{1}, x_{2}, \ldots, x_{n}$ are distinct points in $[0,1]^{r}$,

$$
\sum_{1 \leq i<j \leq n} \frac{1}{\left|x_{i}-x_{j}\right|^{r}} \geq \frac{1}{4 r^{r / 2}} \cdot \frac{9\left(1-2^{-r}\right)}{8 r+9(r+2)\left(1-2^{-r}\right)} \cdot n^{2} \log _{2} n .
$$

In particular, when $r=2$, we deduce that for any $n \in \mathbb{N}$ with $n \geq 2$ and for any distinct points $x_{1}, x_{2}, \ldots, x_{n}$ in $[0,1]^{2}$ we have

$$
\sum_{1 \leq i<j \leq n} \frac{1}{\left|x_{i}-x_{j}\right|^{2}} \geq \frac{27}{1376} \cdot n^{2} \log _{2} n=\frac{27}{1376 \cdot \ln 2} \cdot n^{2} \ln n,
$$

which is a sharpening of Schmidt's original inequality (3.1). More generally, in (3.22) there is a unique value of $c$ which maximises the formula for $C_{r}$. This value of $c$ is in $(0,1)$ and is shown by differentiation to be the solution of the cubic equation

$$
\frac{r \ln 2}{1-2^{-r}} \cdot c=(1-c)^{3} \text {. }
$$

When $r=2$ this solution is moderately close to the value of $1 / 4$ used above to get an explicit value for $C_{2}$ in (3.22).

In the case when $r<2 s$ and $c$ is given in $(0,1)$, a corresponding argument leads to the conclusion that for $r<2 s$, for any $n \in \mathbb{N}$ with $n \geq 2$, and for any distinct points $x_{1}, x_{2}, \ldots, x_{n}$ in $[0,1]^{r}$ we have

$$
\sum_{1 \leq i<j \leq n} \frac{1}{\left|x_{i}-x_{j}\right|^{2 s}} \geq \frac{1}{8 r^{s}} \cdot \frac{2^{2 s}-1}{2^{2(s-r)}} \cdot \frac{c^{2 s / r-1}(1-c)^{2}}{2^{2 s-r}-1+(1-c)^{2}\left(2^{2 s}-1\right) 2^{r-1}} \cdot n^{1+2 s / r} .
$$

The inequalities in Theorem 3.4 may be interpreted as saying something about the average value of the "distinctiveness" of all pairs of $n$ given points in $[0,1]^{r}$. For example, in the case when $r=2$, it follows from (3.23) that

$$
\frac{2}{n(n-1)}\left(\sum_{1 \leq i<j \leq n} \frac{1}{\left|x_{i}-x_{j}\right|^{2}}\right) \geq \frac{27}{688} \log _{2} n .
$$

Here, the left hand side is the average of the terms $\left|x_{i}-x_{j}\right|^{-2}$, and the term $\left|x_{i}-x_{j}\right|^{-2}$ can be taken as some sort of technical measurement of the "distinctiveness" of $x_{i}$ when compared with $x_{j}$ (the smaller this term, the "more different" or the "more distinctive" are $\left.x_{i}, x_{j}\right)$. From this viewpoint, (3.24) says that if there are $n$ distinct points in $[0,1]^{2}$, the average value of the "distinctiveness" of all possible pairs of $n$ points is at least 27/688 times $\log _{2} n$. As a final comment, note that the result for $r=2$ in (ii) of Theorem 3.4, was proved originally by Schmidt [12] in connection with Heilbronn's problem, which is to estimate the supremum of the minimum of the areas of all possible triangles formed from $n$ points in the unit square, where the supremum is taken over all possible distributions of $n$ points. In $n$-dimensions, Heilbronn's problem has been recently studied by G. Barequet [2]. 


\section{Sharpness results in the spaces $\mathcal{D}_{s}\left(L^{2}(\mathbb{R})\right)$}

In this section, a connection is made between the estimates concerning the distribution of points in the $r$-dimensional unit cube, established in Section 3, and sharpness estimates in the spaces $\mathcal{D}_{s}\left(L^{2}(\mathbb{R})\right)$ of Section 2. This type of connection was established originally by Meisters and Schmidt [6], for the case of the circle group $\mathbb{T}$. In the non-compact case, it was shown in $\left[8\right.$, pp.81 and 94] that whereas every function in $\mathcal{D}_{1}\left(L^{2}(\mathbb{R})\right)$ is a sum of 3 first order differences, there are functions in $\mathcal{D}_{1}\left(L^{2}(\mathbb{R})\right)$ which are never equal to a sum of 2 first order differences. However, although it was known that for all $s>0$ every function in $\mathcal{D}_{s}\left(L^{2}(\mathbb{R})\right)$ is a sum of $\operatorname{int}(2 s)+1$ differences of order $s$, the question as to whether there are functions in $\mathcal{D}_{s}\left(L^{2}(\mathbb{R})\right.$ ), which are never the sum of int $(2 s)$ differences of order $s$ has remained open for $s \neq 1$. In this section it is shown that there are many functions in $L^{2}(\mathbb{R})$ which simultaneously belong to all the spaces $\mathcal{D}_{s}\left(L^{2}(\mathbb{R})\right), s>0$ but which cannot be written as a sum of $\operatorname{int}(2 s)$ differences of order $s$ for any $s \geq 1 / 2$. Note that when $0<s<1 / 2$, the question of sharpness in the space $\mathcal{D}_{s}\left(L^{2}(\mathbb{R})\right)$ collapses, for in this case every function in $\mathcal{D}_{s}\left(L^{2}(\mathbb{R})\right)$ is equal to a single difference of order $s$, and this number of differences cannot be reduced. The methods here are based upon some ideas in [8, pp.93-98], but are considerably more general and depend upon the results of the preceding section. Various inequalities are considered in which the formal expression 1/0 may occur. Then, we adopt the convention that $1 / 0=\infty$. Also, we use the notational convention that $x<\infty$ for all $x \in \mathbb{R}$.

Lemma 4.1 Let $r \in \mathbb{N}$, let $I_{1}, I_{2}, \ldots, I_{r}$ and $J$ be compact subsets of $\mathbb{R}$, and let $x_{0} \in J$. Let $f: I_{1} \times I_{2} \times \cdots \times I_{r} \rightarrow \mathbb{R}_{+}$be a continuous function, and let $\epsilon>0$ be given. Then, there is $c>0$ such that the following hold: if $\left(b_{1}, b_{2}, \ldots, b_{r}\right) \in I_{1} \times I_{2} \times \cdots \times I_{r}$, and if $x \in J$ satisfies $\left|x-x_{0}\right|<c$, then

$$
\begin{aligned}
& f\left(b_{1}, b_{2}, \ldots, b_{r}, x_{0}\right)<\frac{\epsilon}{2} \Longrightarrow \frac{1}{f\left(b_{1}, b_{2}, \ldots, b_{r}, x\right)}>\frac{1}{\epsilon}, \quad \text { and } \\
& f\left(b_{1}, b_{2}, \ldots, b_{r}, x_{0}\right) \geq \frac{\epsilon}{2} \quad \Longrightarrow \quad \frac{1}{f\left(b_{1}, b_{2}, \ldots, b_{r}, x\right)}>\frac{1}{2 f\left(b_{1}, b_{2}, \ldots, b_{r}, x_{0}\right)} .
\end{aligned}
$$

Proof. As $I_{1} \times I_{2} \times \cdots \times I_{r} \times J$ is compact, $f$ is uniformly continuous. Hence, there is $c>0$ such that if $\left(b_{1}, b_{2}, \ldots, b_{r}\right) \in I_{1} \times I_{2} \times \cdots \times I_{r}$, and if $x, y \in J$ we have

$$
|x-y|<c \Longrightarrow\left|f\left(b_{1}, b_{2}, \ldots, b_{r}, x\right)-f\left(b_{1}, b_{2}, \ldots, b_{r}, y\right)\right|<\epsilon / 2 .
$$

In particular, if $x \in J$ and $\left|x-x_{0}\right|<c$, and if $f\left(b_{1}, b_{2}, \ldots, b_{r}, x_{0}\right)<\epsilon / 2$, then we have $f\left(b_{1}, b_{2}, \ldots, b_{r}, x\right)<\epsilon$ so that $1 / f\left(b_{1}, b_{2}, \ldots b_{r}, x\right)>1 / \epsilon$.

On the other hand, if $\left|x-x_{0}\right|<c$ and $f\left(b_{1}, b_{2}, \ldots, b_{r}, x_{0}\right) \geq \epsilon / 2$, then we have still that $\left|f\left(b_{1}, b_{2}, \ldots, b_{r}, x\right)-f\left(b_{1}, b_{2}, \ldots, b_{r}, x_{0}\right)\right|<\epsilon / 2$ and so

$$
\left|\frac{f\left(b_{1}, b_{2}, \ldots, b_{r}, x\right)}{f\left(b_{1}, b_{2}, \ldots, b_{r}, x_{0}\right)}-1\right|<\frac{\epsilon}{2} \cdot \frac{1}{f\left(b_{1}, b_{2}, \ldots, b_{r}, x_{0}\right)} \leq \frac{\epsilon}{2} \cdot \frac{2}{\epsilon}=1 .
$$

Hence,

$$
\frac{f\left(b_{1}, b_{2}, \ldots, b_{r}, x\right)}{f\left(b_{1}, b_{2}, \ldots, b_{r}, x_{0}\right)}<2
$$


Lemma 4.2 Let $m \in \mathbb{N}$ and let $a_{1}, \ldots, a_{m}, d_{1}, \ldots, d_{m}$ be complex numbers. Then

$$
\sum_{n=1}^{m} a_{n} d_{n}=\left[\sum_{n=1}^{m-1}\left(a_{n}-a_{n+1}\right)\left(d_{1}+d_{2}+\cdots+d_{n}\right)\right]+a_{m}\left(d_{1}+d_{2}+\cdots+d_{m}\right) .
$$

(When $m=1$, the term in square brackets is taken to be 0 .)

Proof. It can be proved by induction, for example, or from Lemma 3.1.

Lemma 4.3 Let $r \in \mathbb{N}$. Then there is $D_{r}>0$ such that for all $x_{1}, x_{2}, \ldots, x_{r} \in \mathbb{R}$,

$$
\left(\sum_{i=1}^{r} x_{i}^{2}\right)^{r / 2} \geq D_{r} \sum_{i=1}^{r}\left|x_{r}\right|^{r}
$$

Proof. Let $S^{r-1}=\left\{\left(x_{1}, \ldots, x_{r}\right) \in \mathbb{R}^{r}: \sum_{i=1}^{r} x_{i}^{2}=1\right\}$. Then the function given by

$$
\left(x_{1}, \ldots, x_{r}\right) \longmapsto \frac{1}{\sum_{i=1}^{r}\left|x_{i}\right|^{r}}
$$

is continuous and maps $S^{r-1}$ into $(0, \infty)$. As $S^{r-1}$ is compact in $\mathbb{R}^{r}$, this function has a positive minimum $D_{r}$. Then $D_{r}$ has the required property.

Recall that the integer part of a real number $x$ is denoted by int $(x)$. The fractional part of a real number $x$ is denoted by $\mathrm{f} r(x)$. Thus, $x=\operatorname{int}(x)+\mathrm{f} r(x)$ where $\operatorname{int}(x) \in \mathbb{Z}$ and $\mathrm{f} r(x) \in[0,1)$, for all $x \in \mathbb{R}$. If $x \in \mathbb{R}$, let $\mathrm{d}_{\mathbb{Z}}(x)$ denote the distance from $x$ to the nearest integer. Note that

$$
|\mathrm{f} r(x)-\mathrm{f} r(y)| \geq \mathrm{d}_{\mathbb{Z}}(x-y), \text { for all } x, y \in \mathbb{R} .
$$

Lemma 4.4 Let $r \in \mathbb{N}$. Then there is $E_{r}>0$ with the following property: for all $b_{1}, b_{2}, \ldots, b_{r} \in \mathbb{R}$ and all $n \in \mathbb{N}$,

$$
\sum_{j=1}^{n}\left(\sum_{i=1}^{r} \mathrm{~d}_{\mathbb{Z}}\left(j b_{i}\right)^{r}\right)^{-1} \geq E_{r} \cdot n \log _{2} n .
$$

Proof. If $n=1, \log _{2} n=0$, so that it suffices to consider only $n \geq 2$. For each $k \in \mathbb{N}$, let

$$
x_{k}=\left(\mathrm{f} r\left(k b_{1}\right), \mathrm{f} r\left(k b_{2}\right), \ldots, \mathrm{f} r\left(k b_{r}\right)\right) .
$$

Then $x_{k} \in[0,1]^{r}$ for all $k \in \mathbb{N}$. Apply (4.2) and Lemma 4.3 to obtain

$$
\begin{aligned}
\left|x_{k}-x_{\ell}\right|^{r} & =\left(\sum_{i=1}^{r}\left|\mathrm{f} r\left(k b_{i}\right)-\mathrm{f} r\left(\ell b_{i}\right)\right|^{2}\right)^{r / 2} \\
& \geq\left(\sum_{i=1}^{r} \mathrm{~d}_{\mathbb{Z}}\left((k-\ell) b_{i}\right)^{2}\right)^{r / 2} \\
& \geq D_{r}\left(\sum_{i=1}^{r} \mathrm{~d}_{\mathbb{Z}}\left((k-\ell) b_{i}\right)^{r}\right) .
\end{aligned}
$$


Now, let $C_{r}>0$ be chosen so that (ii) of Theorem 3.4 holds. As $k \geq 2$, it now follows that

$$
\sum_{1 \leq k<\ell \leq n}\left(\sum_{i=1}^{r} \mathrm{~d}_{\mathbb{Z}}\left((k-\ell) b_{i}\right)^{r}\right)^{-1} \geq D_{r} \sum_{1 \leq k<\ell \leq n} \frac{1}{\left|x_{k}-x_{\ell}\right|^{r}} \geq C_{r} D_{r} \cdot n^{2} \log _{2} n .
$$

We also have that

$$
\begin{aligned}
\sum_{1 \leq k<\ell \leq n}\left(\sum_{i=1}^{r} \mathrm{~d}_{\mathbb{Z}}\left((k-\ell) b_{i}\right)^{r}\right)^{-1} & =\sum_{j=1}^{n-1} \sum_{\substack{1 \leq k<\ell \leq n \\
k-\ell=j}}\left(\sum_{i=1}^{r} \mathrm{~d}_{\mathbb{Z}}\left(j b_{i}\right)^{r}\right)^{-1} \\
& =\sum_{j=1}^{n-1}(n-j)\left(\sum_{i=1}^{r} \mathrm{~d}_{\mathbb{Z}}\left(j b_{i}\right)^{r}\right)^{-1} \\
& \leq n \sum_{j=1}^{n}\left(\sum_{i=1}^{r} \mathrm{~d}_{\mathbb{Z}}\left(j b_{i}\right)^{r}\right)^{-1} .
\end{aligned}
$$

Using this, we have from (4.3) that

$$
\sum_{j=1}^{n}\left(\sum_{i=1}^{r} \mathrm{~d}_{\mathbb{Z}}\left(j b_{i}\right)^{r}\right)^{-1} \geq n^{-1}\left(\sum_{1 \leq k<\ell \leq n}\left(\sum_{i=1}^{r} \mathrm{~d}_{\mathbb{Z}}\left((k-\ell) b_{i}\right)^{r}\right)^{-1}\right) \geq C_{r} D_{r} n \log _{2} n .
$$

So, the lemma is proved if we put $E_{r}=C_{r} D_{r}$.

Lemma 4.5 Let $r \in \mathbb{N}$ and let $s>0$. Let $\alpha: \mathbb{R} \rightarrow \mathbb{C}$ be a continuous $2 \pi$-periodic function which has an absolutely convergent Fourier series. Assume that there are $c_{1}, c_{2}>0$ such that

$$
c_{1}|x|^{s} \leq|\alpha(x)| \leq c_{2}|x|^{s}, \text { for all } x \in[-\pi, \pi] .
$$

Let $f$ be a function in $L^{2}(\mathbb{R})$. Then $f$ is never equal to a sum of $r$-differences if and only if for all $b_{1}, b_{2}, \ldots, b_{r} \in \mathbb{R}$, we have

$$
\int_{-\infty}^{\infty} \frac{|\widehat{f}(x)|^{2}}{\sum_{i=1}^{r} \mathrm{~d}_{\mathbb{Z}}\left(b_{i} x\right)^{2 s}} d x=\infty .
$$

Proof. By [8, Proposition I.3.5] or by [9, Proposition 3], $f$ is equal to a sum of $r \alpha$-differences if and only if there are $b_{1}, b_{2}, \ldots, b_{r} \in \mathbb{R}$ such that

$$
\int_{-\infty}^{\infty} \frac{|\widehat{f}(x)|^{2}}{\sum_{i=1}^{r}\left|\alpha\left(b_{i} x\right)\right|^{2}} d x<\infty .
$$

But $|x|=2 \pi \mathrm{d}_{\mathbb{Z}}\left(2^{-1} \pi^{-1} x\right)$ for all $x \in[-\pi, \pi]$, and the mapping $x \longmapsto \mathrm{d}_{\mathbb{Z}}\left(2^{-1} \pi^{-1} x\right)$ is $2 \pi$ periodic. So it follows from (4.4) that

$$
c_{1} 2^{s} \pi^{s} \mathrm{~d}_{\mathbb{Z}}\left(2^{-1} \pi^{-1} x\right)^{s} \leq|\alpha(x)| \leq c_{2} 2^{s} \pi^{s} \mathrm{~d}_{\mathbb{Z}}\left(2^{-1} \pi^{-1} x\right)^{s}, \quad \text { for all } \quad x \in \mathbb{R} .
$$


Hence, we deduce from (4.4) and (4.6) that $f$ is equal to a sum of $r \alpha$-differences if and only if there are $b_{1}, b_{2}, \ldots, b_{r} \in \mathbb{R}$ such that

$$
\int_{-\infty}^{\infty} \frac{|\widehat{f}(x)|^{2}}{\sum_{i=1}^{r} \mathrm{~d}_{\mathbb{Z}}\left(2^{-1} \pi^{-1} b_{i} x\right)^{2 s}} d x<\infty
$$

This is clearly equivalent to (4.5).

For $r \in \mathbb{N}$ the function

$$
\left(b_{1}, b_{2}, \ldots, b_{r}, x\right) \longmapsto \sum_{i=1}^{r} \mathrm{~d}_{\mathbb{Z}}\left(b_{i} x\right)^{r},
$$

is continuous and maps $\mathbb{R}^{r+1}$ into $\mathbb{R}_{+}$. So, if $n \in \mathbb{N}$ we can apply Lemma 4.1 simultaneously to the first $n$ of the functions given by $(4.7)$ for $r=1,2, \ldots, n$. It follows that for each $n \in \mathbb{N}$ with $n \geq 2$ there is $c_{n}$ satisfying $0<c_{n}<1 / 4$ such that the following holds: for any $x$ in $\left[n-c_{n}, n+c_{n}\right]$, for any $r \in\{1,2, \ldots, n\}$, and for any $b_{1}, b_{2}, \ldots, b_{r}$ in $\mathbb{R}$ with $\left|b_{1}\right| \leq n$, $\left|b_{2}\right| \leq n, \ldots,\left|b_{r}\right| \leq n$, we have

$$
\begin{aligned}
& \sum_{j=1}^{r} \mathrm{~d}_{\mathbb{Z}}\left(b_{j} n\right)^{r}<\frac{1}{2 n^{2}\left(\log _{2} n\right)^{2}} \Longrightarrow \frac{1}{\sum_{j=1}^{r} \mathrm{~d}_{\mathbb{Z}}\left(b_{j} x\right)^{r}}>n^{2}\left(\log _{2} n\right)^{2}, \quad \text { and } \\
& \sum_{j=1}^{r} \mathrm{~d}_{\mathbb{Z}}\left(b_{j} n\right)^{r} \geq \frac{1}{2 n^{2}\left(\log _{2} n\right)^{2}} \quad \Longrightarrow \quad \frac{1}{\sum_{j=1}^{r} \mathrm{~d}_{\mathbb{Z}}\left(b_{j} x\right)^{r}}>\frac{1}{2 \sum_{j=1}^{r} \mathrm{~d}_{\mathbb{Z}}\left(b_{j} n\right)^{r}} .
\end{aligned}
$$

For later use, note that in $(4.8), c_{n}$ may be chosen to be as small as we wish it to be. Hence we can always choose the sequence $\left(c_{n}\right)$ so that

$$
\sum_{n=2}^{\infty} \frac{\sqrt{c_{n}}}{\sqrt{n} \log _{2} n}<\infty .
$$

Now, for a subinterval $J$ of $\mathbb{R}$, let $\lambda(J)$ denote its length. Let $\Theta$ be a function with domain $\mathbb{N}$ such that $\Theta(n)$ is a subinterval of positive length of $\left[n-c_{n}, n+c_{n}\right]$ for each $n \in \mathbb{N}$. Note that $\Theta(n)$ may also be denoted by $\Theta_{n}$. The idea is that for each such $\Theta$ we define a function $f_{\Theta}$ in $L^{2}(\mathbb{R})$ which we shall see is the sum of $\operatorname{int}(2 s)+1$ differences of order $s$ but is never the sum of $\operatorname{int}(2 s)$ differences of order $s$. Note that the manner in which the $c_{n}$ have been chosen ensures that the intervals $\left[n-c_{n}, n+c_{n}\right]$ for $n=2,3,4, \ldots$ are disjoint, so that the intervals $\Theta(n)$ for $n=2,3, \ldots$ are also disjoint. The function $f_{\Theta}$ in $L^{2}(\mathbb{R})$ is given via its Fourier transform $\widehat{f}_{\Theta}$ by requiring that

$$
\widehat{f}_{\Theta}(x)= \begin{cases}\frac{1}{\sqrt{n \lambda(\Theta(n))} \log _{2} n}, & \text { if } x \in \Theta(n) \text { and } n \geq 2 ; \\ 0, & \text { if } x \notin \bigcup_{n=2}^{\infty} \Theta(n) .\end{cases}
$$


Note that in (4.10), the definition we use for the Fourier transform $\widehat{f}$ of a function $f$ in $L^{2}(\mathbb{R})$ is based upon the following definition for a function $g$ in $L^{1}(\mathbb{R})$ :

$$
\widehat{g}(x)=\frac{1}{\sqrt{2 \pi}} \int_{-\infty}^{\infty} g(y) e^{-i x y} d y, \text { for } x \in \mathbb{R} .
$$

Now, it follows from (4.10) that

$$
\int_{-\infty}^{\infty}\left|\widehat{f}_{\Theta}(x)\right|^{2} d x=\sum_{n=2}^{\infty} \frac{1}{n\left(\log _{2} n\right)^{2}}<\infty
$$

so that $\widehat{f}_{\Theta}$ is in $L^{2}(\mathbb{R})$. By Plancherel's Theorem, there is $f_{\Theta} \in L^{2}(\mathbb{R})$ whose Fourier transform is the given function $\widehat{f}_{\Theta}$. Now, by $(4.10)$ we see that the Fourier transform of $f_{\Theta}$ vanishes in a neighbourhood of the origin, so it follows immediately from Theorem 2.1 that the function $f_{\Theta}$ is in every space $\mathcal{D}_{s}\left(L^{2}(\mathbb{R})\right), s>0$. So, by Theorem 2.2 the function $f_{\Theta}$ is a sum of $\operatorname{int}(2 s)+1$ differences of order $s$ for each $s \in \mathbb{N}$. However, for no $s \in \mathbb{N}$ is $f_{\Theta}$ ever a sum of int $(2 s)$ differences of order $s$, and the following result is crucial in establishing this fact.

Proposition 4.6 Let $\left(c_{n}\right)$ be a sequence in $(0, \infty)$, chosen so as to ensure that $(4.8)$ holds, and let $\Theta$ be a function with domain $\mathbb{N}$ such that $\Theta(n)$ is a subinterval of positive length of $\left[n-c_{n}, n+c_{n}\right]$ for all $n \in \mathbb{N}$. Let $f_{\Theta}$ be the function in $L^{2}(\mathbb{R})$ whose Fourier transform $\widehat{f}_{\Theta}$ is given by

$$
\widehat{f}_{\Theta}=\sum_{n=2}^{\infty} \frac{1}{\sqrt{n \lambda(\Theta(n))} \log _{2} n} \chi_{\Theta(n)},
$$

as described alternatively in (4.10). Then

$$
f_{\Theta} \in \bigcap_{s>0} \mathcal{D}_{s}\left(L^{2}(\mathbb{R})\right)
$$

and for any $A \in(0, \infty), r \in \mathbb{N}$ and $b_{1}, b_{2}, \ldots, b_{r}$ in $\mathbb{R}$,

$$
\int_{A}^{\infty} \frac{\left|\widehat{f}_{\Theta}(x)\right|^{2}}{\sum_{j=1}^{r} \mathrm{~d}_{\mathbb{Z}}\left(b_{j} x\right)^{r}} d x=\infty
$$

Proof. As $\Theta_{n} \subseteq\left[n-c_{n}, n+c_{n}\right]$, and as $0<c_{n}<1 / 4$, we see from (4.11) that $\widehat{f}_{\Theta}(x)=0$ for all $x \in[-1 / 2,1 / 2]$. So, by Theorem 2.1, we have $f \in \bigcap_{s>0} \mathcal{D}_{s}\left(L^{2}(\mathbb{R})\right)$, which proves (4.12).

Now let $b_{1}, b_{2}, \ldots, b_{r}$ be given in $\mathbb{R}$ and let $A>0$ be given. We prove that (4.13) holds. To this end, choose $m \in \mathbb{N}$ such that $2 \leq m, r \leq m, \Theta(m) \subseteq[A, \infty)$ and $\left|b_{1}\right| \leq m,\left|b_{2}\right| \leq$ $m, \ldots,\left|b_{r}\right| \leq m$. Then,

$$
\int_{A}^{\infty} \frac{\left|\widehat{f}_{\Theta}(x)\right|^{2}}{\sum_{i=1}^{r} \mathrm{~d}_{\mathbb{Z}}\left(b_{i} x\right)^{r}} d x \geq \int_{\cup_{n=m}^{\infty} \Theta_{n}} \frac{\left|\widehat{f}_{\Theta}(x)\right|^{2}}{\sum_{i=1}^{r} \mathrm{~d}_{\mathbb{Z}}\left(b_{i} x\right)^{r}} d x
$$




$$
\begin{aligned}
& =\sum_{n=m}^{\infty} \int_{\Theta_{n}} \frac{\left|\widehat{f}_{\Theta}(x)\right|^{2}}{\sum_{i=1}^{r} \mathrm{~d}_{\mathbb{Z}}\left(b_{i} x\right)^{r}} d x \\
& =\sum_{n=m}^{\infty} \frac{1}{n \lambda\left(\Theta_{n}\right)\left(\log _{2} n\right)^{2}} \int_{\Theta_{n}} \frac{1}{\sum_{i=1}^{r} \mathrm{~d}_{\mathbb{Z}}\left(b_{i} x\right)^{r}} d x .
\end{aligned}
$$

Let, for $n=m, m+1, m+2, \ldots$,

$$
\mu_{n}=\inf \left\{\left(\sum_{i=1}^{r} \mathrm{~d}_{\mathbb{Z}}\left(b_{i} x\right)^{r}\right)^{-1}: x \in \Theta(n)\right\} .
$$

Note that this infimum is finite because $\sum_{i=1}^{r} \mathrm{~d}_{\mathbb{Z}}\left(b_{i} x\right)^{r}$ is strictly positive for some $x$ in $\Theta(n)$. Also, as $\mathrm{d}_{\mathbb{Z}}(x) \in[0,1 / 2]$, it is clear that $\mu_{n} \geq r^{-1} 2^{r}$. So, let us note incidentally that $\mu_{n} \in\left(r^{-1} 2^{r}, \infty\right)$. It follows from (4.14) and the definition of $\mu_{n}$ that

$$
\int_{A}^{\infty} \frac{\left|\widehat{f}_{\Theta}(x)\right|^{2}}{\sum_{i=1}^{r} \mathrm{~d}_{\mathbb{Z}}\left(b_{i} x\right)^{r}} d x \geq \sum_{n=m}^{\infty} \frac{\mu_{n}}{n\left(\log _{2} n\right)^{2}} .
$$

Now, observe that for $n \geq 2$,

$$
\begin{aligned}
\frac{1}{n\left(\log _{2} n\right)^{2}}-\frac{1}{(n+1) \log _{2}(n+1)^{2}} & \geq \frac{1}{\left(\log _{2}(n+1)\right)^{2}}\left(\frac{1}{n}-\frac{1}{n+1}\right) \\
& \geq \frac{1}{(n+1)^{2}\left(\log _{2}(n+1)\right)^{2}} \\
& \geq \frac{1}{9} \cdot \frac{1}{n^{2}\left(\log _{2} n\right)^{2}} .
\end{aligned}
$$

Using Lemma 4.2, (4.15) and (4.16), we now have

$$
\begin{aligned}
\int_{A}^{\infty} \frac{\left|\widehat{f}_{\Theta}(x)\right|^{2}}{\sum_{i=1}^{r} \mathrm{~d}_{\mathbb{Z}}\left(b_{i} x\right)^{r}} d x & \geq \sum_{n=m}^{\infty} \frac{\mu_{n}}{n\left(\log _{2} n\right)^{2}} \\
& \geq \sum_{n=m}^{\infty}\left(\frac{1}{n\left(\log _{2} n\right)^{2}}-\frac{1}{(n+1)\left(\log _{2}(n+1)^{2}\right.}\right) \cdot\left(\sum_{j=m}^{n} \mu_{j}\right) \\
& \geq \frac{1}{9} \sum_{n=m}^{\infty} \frac{\sum_{j=m}^{n} \mu_{j}}{n^{2}\left(\log _{2} n\right)^{2}} .
\end{aligned}
$$

There are two cases to consider in (4.17).

$C A S E I$. Assume that there are $n_{1}, n_{2}, \ldots$ in $\mathbb{N}$ such that $m \leq n_{1}<n_{2}<n_{3}<\cdots$ and for all $\ell=1,2, \ldots$,

$$
\sum_{i=1}^{r} \mathrm{~d}_{\mathbb{Z}}\left(b_{i} n_{\ell}\right)^{r}<\frac{1}{2 n_{\ell}^{2}\left(\log _{2} n_{\ell}\right)^{2}}
$$


Then, as $r \leq m$ and $r \in\left\{1,2, \ldots, n_{\ell}\right\}$ for all $\ell$, and as $\left|b_{i}\right| \leq m$ for $i=1,2, \ldots, r$, we may apply (4.8) to obtain

$$
\mu_{n_{\ell}} \geq n_{\ell}^{2}\left(\log _{2} n_{\ell}\right)^{2}, \text { for all } \ell=1,2, \ldots
$$

It follows from this, together with (4.17), that

$$
\begin{aligned}
\int_{A}^{\infty} \frac{\left|\widehat{f}_{\Theta}(x)\right|^{2}}{\sum_{i=1}^{r} \mathrm{~d}_{\mathbb{Z}}\left(b_{i} x\right)^{r}} d x & \geq \frac{1}{9} \sum_{\ell=1}^{\infty} \frac{\sum_{j=m}^{n_{\ell}} \mu_{j}}{n_{\ell}{ }^{2}\left(\log _{2} n_{\ell}\right)^{2}} \\
& \geq \frac{1}{9} \sum_{\ell=1}^{\infty} \frac{\mu_{n_{\ell}}}{n_{\ell}{ }^{2}\left(\log _{2} n_{\ell}\right)^{2}} \\
& \geq \frac{1}{9} \sum_{\ell=1}^{\infty} 1 \\
& =\infty .
\end{aligned}
$$

$C A S E$ II. When Case I does not occur, there is $m_{0} \geq m$ such that for all $n \geq m_{0}$,

$$
\sum_{i=1}^{r} \mathrm{~d}_{\mathbb{Z}}\left(b_{i} n\right)^{r} \geq \frac{1}{2 n^{2}\left(\log _{2} n\right)^{2}} .
$$

It follows from (4.8) and (4.19) that for all $n \geq m_{0}$,

$$
\mu_{n} \geq \frac{1}{2 \sum_{i=1}^{r} \mathrm{~d}_{\mathbb{Z}}\left(b_{i} n\right)^{r}} .
$$

Also, (4.19) implies that

$$
\sum_{i=1}^{r} \mathrm{~d}_{\mathbb{Z}}\left(b_{i} n\right)^{r}>0, \text { for all } n=1,2,3, \ldots
$$

This is so because, otherwise, we would have $\sum_{i=1}^{r} \mathrm{~d}_{\mathbb{Z}}\left(b_{i} \ell\right)^{r}=0$ for some $\ell \in\left\{1,2, \ldots, m_{0}-1\right\}$ and this would imply that $\sum_{i=1}^{r} \mathrm{~d}_{\mathbb{Z}}\left(b_{i} \ell j\right)^{r}=0$ for all $j=1,2,3 \ldots$, contrary to (4.19). Now, put

$$
C\left(b_{1}, \ldots, b_{r}, m_{0}\right)=\left(\sum_{j=1}^{m_{0}-1} \frac{1}{\sum_{i=1}^{r} \mathrm{~d}_{\mathbb{Z}}\left(b_{i} j\right)^{r}}\right) .
$$

Note that $(4.21)$ implies that $C\left(b_{1}, \ldots, b_{r}, m_{0}\right) \in(0, \infty)$. Using Lemma 4.4, (4.20), (4.21) and (4.22), it now follows from (4.17) that

$$
\int_{A}^{\infty} \frac{\left|\widehat{f}_{\Theta}(x)\right|^{2}}{\sum_{i=1}^{r} \mathrm{~d}_{\mathbb{Z}}\left(b_{i} x\right)^{r}} d x \geq \frac{1}{9} \sum_{n=m_{0}}^{\infty} \frac{\sum_{j=m_{0}}^{n} \mu_{j}}{n^{2}\left(\log _{2} n\right)^{2}}
$$




$$
\begin{aligned}
& \geq \frac{1}{9} \sum_{n=m_{0}}^{\infty} \frac{1}{n^{2}\left(\log _{2} n\right)^{2}} \cdot \frac{1}{2}\left(\sum_{j=m_{0}}^{n} \frac{1}{\sum_{i=1}^{r} \mathrm{~d}_{\mathbb{Z}}\left(b_{i} j\right)^{r}}\right) \\
& =\frac{1}{18} \sum_{n=m_{0}}^{\infty} \frac{1}{n^{2}\left(\log _{2} n\right)^{2}} \cdot\left(\sum_{j=1}^{n} \frac{1}{\sum_{i=1}^{r} \mathrm{~d}_{\mathbb{Z}}\left(b_{i} j\right)^{r}}-\sum_{j=1}^{m_{0}-1} \frac{1}{\left.\sum_{i=1}^{r} \mathrm{~d}_{\mathbb{Z}}\left(b_{i} j\right)\right)^{r}}\right) \\
& \geq \frac{1}{18} \sum_{n=m_{0}}^{\infty} \frac{1}{n^{2}\left(\log _{2} n\right)^{2}} \cdot\left(E_{r} n \log _{2} n-C\left(b_{1}, \ldots, b_{r}, m_{0}\right)\right) \\
& =\frac{1}{18} \sum_{n=m_{0}}^{\infty} \frac{1}{n \log _{2} n} \cdot\left(E_{r}-\frac{C\left(b_{1}, \ldots, b_{r}, m_{0}\right)}{n \log _{2} n}\right)
\end{aligned}
$$

Now, $b_{1}, \ldots, b_{r}$ are given, and once $m_{0}$ has subsequently been chosen in accordance with Case II, $C\left(b_{1}, \ldots, b_{r}, m_{0}\right)$ is constant, so that it follows from (4.23) that there is $q \in \mathbb{N}$ with $q>m_{0}$ such that

$$
\int_{A}^{\infty} \frac{\left|\widehat{f}_{\Theta}(x)\right|^{2}}{\sum_{i=1}^{r} \mathrm{~d}_{\mathbb{Z}}\left(b_{i} x\right)^{r}} d x \geq \frac{E_{r}}{36}\left(\sum_{n=q+1}^{\infty} \frac{1}{n \log _{2} n}\right)=\infty .
$$

It now follows from (4.18) and (4.24) that the conclusion (4.13) holds, as required.

It is perhaps of interest to point out that if the estimate for $\mu_{k}$ mentioned in the proof, namely $\mu_{k} \geq r^{-1} 2^{r}$, is placed directly into (4.17), then the resulting series on the right hand side of (4.17) is convergent. This is an indication of the delicacy of the argument which is required to show that in fact the series on the right hand side of (4.17) is divergent.

The Fourier Inversion Theorem says that the Fourier transform of $\widehat{f}$ evaluated (maybe almost everywhere) at $-x$ is the function $x \mapsto f(x)$. This is used in establishing the following result, which identifies more explicitly the function $f_{\Theta}$ of Proposition 4.6, and derives properties of $f_{\Theta}$ related to questions of sharpness in the spaces $\mathcal{D}_{s}\left(L^{2}(\mathbb{R})\right), s>0$..

Theorem 4.7 Let $f_{\Theta}$ be the function as described in (4.11), or alternatively in (4.12). Let $a_{n}$ be the midpoint of the interval $\Theta(n)$ for all $n \in \mathbb{N}$. Then the following hold.

(i) $f_{\Theta}$ is equal to the sum of the following series which is convergent in $L^{2}(\mathbb{R})$.

$$
f_{\Theta}(x)=\frac{1}{\sqrt{2 \pi}} \sum_{n=2}^{\infty} \frac{\sqrt{\lambda\left(\Theta_{n}\right)}}{\sqrt{n} \log _{2} n} \cdot \frac{\sin \left[\lambda\left(\Theta_{n}\right) x / 2\right]}{\left[\lambda\left(\Theta_{n}\right) x / 2\right]} \cdot \exp \left(i a_{n} x\right) .
$$

(ii) Let $s \in(0, \infty)$ with $s \geq 1 / 2$, and let $\alpha: \mathbb{R} \longrightarrow \mathbb{C}$ be a $2 \pi$-periodic continuous function such that for some $c_{1}, c_{2}>0$,

$$
c_{1}|x|^{s} \leq|\alpha(x)| \leq c_{2}|x|^{s}, \text { for all } x \in[-\pi, \pi]
$$


Then $f_{\Theta}$ is equal to a sum of $\operatorname{int}(2 s)+1 \alpha$-differences, but $f$ is never the sum of int $(2 s)$ $\alpha$-differences.

(iii) The sequence $\Theta$, used to define $f_{\Theta}$ in $(4.9)$, may be chosen so that $\sum_{n=2}^{\infty} \frac{\sqrt{\lambda\left(\Theta_{n}\right)}}{\sqrt{n} \log _{2} n}<$ $\infty$, in which case $f_{\Theta}$ is also in the space $C_{0}(\mathbb{R})$ of continuous complex valued functions on $\mathbb{R}$ which vanish at $\infty$.

Proof. The function $\chi_{\Theta(n)}$ is the Fourier transform of the function

$$
x \longmapsto \frac{\lambda\left(\Theta_{n}\right)}{\sqrt{2} \pi} \exp \left(\sqrt{-1} a_{n} x\right)\left(\frac{\sin \left[\lambda\left(\Theta_{n}\right) x / 2\right]}{[x / 2]}\right) .
$$

Now the series in (4.11), which defines the function $\widehat{f}_{\Theta}$, is convergent in $L^{2}(\mathbb{R})$ because $\sum_{n=2}^{\infty} 1 / n\left(\log _{2} n\right)^{2}<\infty$ and because the functions $\lambda\left(\Theta_{n}\right)^{-1 / 2} \chi_{\Theta(n)}$ for $n=2,3, \ldots$ are orthonormal as their supports do not overlap. Taking inverse Fourier transforms in (4.11) therefore establishes statement (i).

To prove (ii), first observe that because the function $f_{\Theta}$ is in $\mathcal{D}_{s}(\mathbb{R})$, it is a sum of $\operatorname{int}(2 s)+1 \alpha$-differences by Theorem 2.2. However, from (4.13) in Proposition 4.6 we have

$$
\int_{-\infty}^{\infty} \frac{\left|\widehat{f}_{\Theta}(x)\right|^{2}}{\sum_{i=1}^{\operatorname{int}(2 s)} \mathrm{d}_{\mathbb{Z}}\left(b_{i} x\right)^{\operatorname{int}(2 s)}} d x=\infty
$$

and it follows from Lemma 4.5 that $f_{\Theta}$ is never the sum of int $(2 s) \alpha$-differences, so that (ii) holds.

Finally, the fact that the sequence $\Theta$ may be chosen so that $\sum_{n=2}^{\infty} \frac{\sqrt{\lambda\left(\Theta_{n}\right)}}{\sqrt{n} \log _{2} n}<\infty$ follows from (4.9). In this case we see that the series in (4.25) is uniformly convergent in $C_{0}(\mathbb{R})$, and hence the sum belongs to $C_{0}(\mathbb{R})$. This proves (iii).

Theorem 4.8 There is a vector subspace $V$ of $L^{2}(\mathbb{R})$ which has the following properties.

(i) $V \subseteq \bigcap_{s>0} \mathcal{D}_{s}\left(L^{2}(\mathbb{R})\right)$.

(ii) If $s \geq 1 / 2$, and if $\alpha: \mathbb{R} \longrightarrow \mathbb{C}$ is any $2 \pi$-periodic continuous function such that for some $c_{1}, c_{2}>0$,

$$
c_{1}|x|^{s} \leq|\alpha(x)| \leq c_{2}|x|^{s}, \text { for all } x \in[-\pi, \pi],
$$

then any non-zero function $f$ in $V$ is a sum of $\operatorname{int}(2 s)+1 \alpha$-differences, but $f$ is never the sum of int $(2 s) \alpha$-differences.

(iii) The algebraic dimension of $V$ is the cardinality of the continuum.

(iv) The subspace $V$ may be chosen so that in addition to having the above properties it is a subspace of $C_{0}(\mathbb{R}) \cap L^{2}(\mathbb{R})$.

Proof. Let $\Gamma$ be the set of all functions $\gamma: \mathbb{N} \longrightarrow \mathbb{N}$ such that for all $n \in \mathbb{N}$,

$$
\gamma(n) \in\left\{1,2, \ldots, 2^{n}\right\} \text { and } \gamma(n+1) \in\{2 \gamma(n)-1,2 \gamma(n)\} .
$$


The cardinality of $\Gamma$ is that of the continuum, and it can be seen that if $\gamma_{1}, \gamma_{2} \in \Gamma$ with $\gamma_{1} \neq \gamma_{2}$, then there is $A \in \mathbb{N}$ such that

$$
\gamma_{1}(n) \neq \gamma_{2}(n) \text { for all } n \geq A \text {. }
$$

Now, for each $n \in \mathbb{N}$ suppose that $J_{n, 1}, J_{n, 2}, \ldots, J_{n, 2^{n}}$ are subintervals of $\mathbb{R}$ having positive lengths such that

$$
\begin{gathered}
J_{n, k} \cap J_{n, \ell}=\emptyset \text { for all } k, \ell \in\left\{1,2, \ldots, 2^{n}\right\} \text { with } k \neq \ell, \text { and } \\
\qquad \bigcup_{j=1}^{2^{n}} J_{n, j} \subseteq\left[n-c_{n}, n+c_{n}\right] .
\end{gathered}
$$

Now, as $0<c_{n}<1 / 4$ for all $n$, the intervals $\left[n-c_{n}, n+c_{n}\right]$ for $n=1,2, \ldots$ are pairwise disjoint. For each $\gamma \in \Gamma$ define $\Theta_{\gamma}$ on $\mathbb{N}$ by

$$
\Theta_{\gamma}(n)=J_{n, \gamma(n)}, \text { for all } n \in \mathbb{N} .
$$

Then, for each $\gamma \in \Gamma$, the sequence of intervals $\Theta_{\gamma}$ is of the type used in (4.10) and (4.11) to define the function $f_{\Theta}$. In fact, by (4.11) we have that for each $\gamma \in \Gamma$ the function $f_{\Theta_{\gamma}} \in L^{2}(\mathbb{R})$ is given by

$$
\widehat{f}_{\Theta_{\gamma}}=\sum_{n=2}^{\infty} \frac{1}{\sqrt{n \lambda\left(\Theta_{\gamma}(n)\right)} \log _{2} n} \chi_{\Theta_{\gamma}(n)} .
$$

We let $V$ be the vector space finitely spanned by the functions $f_{\Theta_{\gamma}}, \gamma \in \Gamma$. Then it follows from (4.12) that $V \subseteq \bigcap_{s>0} \mathcal{D}_{s}\left(L^{2}(\mathbb{R})\right)$, which proves (i).

In order to prove (iii), it suffices to observe that the family of functions $\left\{f_{\Theta_{\gamma}}: \gamma \in \Gamma\right\}$ is uncountable and to prove that any finite subset of this family is linearly independent. Let $\gamma_{1}, \gamma_{2}, \ldots, \gamma_{q}$ be distinct elements of $\Gamma$. By (4.26) there is $A \in \mathbb{N}$ such that

$$
k>A \text { and } i, j \in\{1,2, \ldots, q\} \text { with } i \neq j \Longrightarrow \gamma_{i}(k) \neq \gamma_{j}(k) .
$$

It now follows from (4.27), (4.28) and (4.29) that if $i, j \in\{1,2, \ldots, q\}$ with $i \neq j$, we have

$$
\left(\bigcup_{k=A+1}^{\infty} \Theta_{k, \gamma_{i}(k)}\right) \bigcap\left(\bigcup_{k=A+1}^{\infty} \Theta_{k, \gamma_{j}(k)}\right)=\emptyset .
$$

Then, from (4.30), (4.31) and (4.32) we see that if $i, j \in\{1,2, \ldots, q\}$ with $i \neq j$,

$$
\widehat{f}_{\Theta_{\gamma_{i}}}(x) \widehat{f}_{\Theta_{\gamma_{j}}}(x)=0, \text { for all } x \in[A, \infty) .
$$

Now, let $a_{1}, a_{2}, \ldots, a_{q}$ be non-zero elements in $\mathbb{C}$, let $r \in \mathbb{N}$ and let $b_{1}, b_{2}, \ldots, b_{r} \in \mathbb{R}$. Then, (4.33) now gives

$$
\int_{-\infty}^{\infty} \frac{\left|\sum_{k=1}^{q} a_{k} \widehat{f}_{\Theta_{\gamma_{k}}}(x)\right|^{2}}{\sum_{i=1}^{r} \mathrm{~d}_{\mathbb{Z}}\left(b_{i} x\right)^{r}} d x \geq \int_{A}^{\infty} \frac{\left|\sum_{k=1}^{q} a_{k} \widehat{f}_{\Theta_{\gamma_{k}}}(x)\right|^{2}}{\sum_{i=1}^{r} \mathrm{~d}_{\mathbb{Z}}\left(b_{i} x\right)^{r}} d x
$$




$$
\begin{aligned}
& =\int_{A}^{\infty} \frac{\sum_{k=1}^{q}\left|a_{k}\right|^{2}\left|\widehat{f}_{\Theta_{\gamma_{k}}}(x)\right|^{2}}{\sum_{i=1}^{r} \mathrm{~d}_{\mathbb{Z}}\left(b_{i} x\right)^{r}} d x \\
& =\sum_{k=1}^{q}\left|a_{k}\right|^{2} \int_{A}^{\infty} \frac{\left|\widehat{f}_{\Theta_{\gamma_{k}}}(x)\right|^{2}}{\sum_{i=1}^{r} \mathrm{~d}_{\mathbb{Z}}\left(b_{i} x\right)^{r}} d x \\
& =\infty,
\end{aligned}
$$

where (4.13) in Proposition 4.6 has been used with $\Theta_{\gamma_{k}}$ in place of $\Theta$. We deduce certain facts from (4.34). First, $\sum_{k=1}^{q} a_{k} f_{\Theta_{\gamma_{k}}} \neq 0$, and it follows that the family of functions $\left\{f_{\Theta_{\gamma}}: \gamma \in \Gamma\right\}$ is linearly independent. This implies that the space $V$ has algebraic dimension equal to that of the continuum, which establishes (iii). However, we also conclude from Lemma 4.5 that for $s \geq 1 / 2$ no non-zero function in $V$ can be expressed as a sum of int $(2 s) \alpha$-differences, if $\alpha$ is a function as given in statement (ii). But we know from (i) and Theorem 2.2 that every function in $V$ is a sum of $\operatorname{int}(2 s)+1 \alpha$-differences. This proves (ii).

\section{$5 \quad$ Results in the spaces $\mathcal{F}_{p}(\mathbb{R}), 1 \leq p<\infty$}

The space $L^{2}(\mathbb{R})$ has been the one in which the results of this paper so far have been presented. In this section, we show how the results in $L^{2}(\mathbb{R})$ may be extended to certain Banach spaces of distributions over $\mathbb{R}$. Let $1 \leq p<\infty$. Then $\mathcal{F}_{p}(\mathbb{R})$ is defined to be the space of tempered distributions whose Fourier transforms are in $L^{p}(\mathbb{R})$, and $\mathcal{F}_{p}(\mathbb{R})$ is a Banach space in the norm $\||\cdot|\|_{p}$, where

$$
\|\mid f\|_{p}=\|\widehat{f}\|_{p}, \text { for all } f \in \mathcal{F}_{p}(\mathbb{R}) .
$$

For the circle group, the $\mathcal{F}_{p}$ spaces are discussed in [6], and for locally compact abelian groups see $\left[8\right.$, II.2]. By Plancherel's Theorem, $\mathcal{F}_{2}(\mathbb{R})$ may be identified with the space $L^{2}(\mathbb{R})$. The results already obtained in the context of $L^{2}(\mathbb{R})$ are now extended to the spaces $\mathcal{F}_{p}(\mathbb{R})$. Let $s>0$ and let $\alpha: \mathbb{R} \rightarrow \mathbb{C}$ be a continuous, $2 \pi$-periodic function which has an absolutely convergent Fourier series. Then, by analogy with the definition given in Section 2 for functions in $L^{2}(\mathbb{R})$, a distribution $f$ in $\mathcal{F}_{p}(\mathbb{R})$ is called an $\alpha$-difference if there are $y \in \mathbb{R}$ and $g \in \mathcal{F}_{p}(\mathbb{R})$ such that

$$
f=\sum_{k=-\infty}^{\infty} \widehat{\alpha}(k) \delta_{-k y} * g .
$$

The following Theorem is analogous to Theorem 2.1. A proof may be found in $[7$, Theorem 2] or [8, Proposition II.5.2].

Theorem 5.1 Let $1 \leq p<\infty$, let $s \in(0, \infty)$ and let $\alpha: \mathbb{R} \longrightarrow \mathbb{C}$ be a continuous $2 \pi$ periodic function which has an absolutely convergent Fourier series and for which there are $c_{1}, c_{2}>0$ such that $c_{1}|x|^{s} \leq|\alpha(x)| \leq c_{2}|x|^{s}$ for all $x \in[-\pi, \pi]$. Let $\mathcal{D}_{\alpha}\left(\mathcal{F}_{p}(\mathbb{R})\right)$ denote the 
vector space consisting of all distributions in $\mathcal{F}_{p}(\mathbb{R})$ which are finite sums of $\alpha$-differences. Then,

$$
\left\{\widehat{g}: g \in \mathcal{D}_{\alpha}\left(\mathcal{F}_{p}(\mathbb{R})\right)\right\}=L^{p}\left(\mathbb{R},\left(1+\frac{1}{|x|^{p s}}\right) d x\right) .
$$

Theorem 5.1 shows that the space $\mathcal{D}_{\alpha}\left(\mathcal{F}_{p}(\mathbb{R})\right)$ depends upon $s$ but not upon $\alpha$. So, the space $\mathcal{D}_{\alpha}\left(\mathcal{F}_{p}(\mathbb{R})\right)$ is denoted by $\mathcal{D}_{s}\left(\mathcal{F}_{p}(\mathbb{R})\right)$. Also, for any $s>0$, as in the $L^{2}(\mathbb{R})$ case, there is a function $\alpha$ satisfying the assumptions in Theorem 5.1. Hence, we see that the space $\mathcal{D}_{s}\left(\mathcal{F}_{p}(\mathbb{R})\right)$ is defined for all $1 \leq p<\infty$ and all $s>0$. The following theorem is the result of Theorem 5.1 and these remarks, together with results concerning the spaces $\mathcal{D}_{s}\left(\mathcal{F}_{p}(\mathbb{R})\right)$ which may be found in $[7,8]$, as above.

Theorem 5.2 Let $1 \leq p<\infty$ and let $s>0$. Then, $\mathcal{D}_{s}\left(\mathcal{F}_{p}(\mathbb{R})\right)$ is a Banach space in the norm $\||\cdot|\|_{p, s}$ given by

$$
\||f|\|_{p, s}=\int_{-\infty}^{\infty}|\widehat{f}(x)|^{p}\left(1+\frac{1}{|x|^{p s}}\right) d x .
$$

Also, each element of $\mathcal{D}_{s}\left(\mathcal{F}_{p}(\mathbb{R})\right)$ is a sum of $\operatorname{int}(p s)+1 \alpha$-differences.

The aim in this section is to show that Theorem 4.8 , obtained in the space $L^{2}(\mathbb{R})$, has an analogue in the spaces $\mathcal{F}_{p}(\mathbb{R}), 1 \leq p<\infty$. In particular, it is a consequence of Theorem 5.4 below that when $s \geq 1 / p$, the estimate of int $(p s)+1$ as the required number of $\alpha$-differences in Theorem 5.2 is sharp.

Lemma 5.3 Let $1 \leq p<\infty$, let $s \in(0, \infty)$ and let $r \in \mathbb{N}$. Let $\alpha: \mathbb{R} \longrightarrow \mathbb{C}$ be a continuous $2 \pi$-periodic function which has an absolutely convergent Fourier series and for which there are $c_{1}, c_{2}>0$ such that $c_{1}|x|^{s} \leq|\alpha(x)| \leq c_{2}|x|^{s}$ for all $x \in[-\pi, \pi]$. Let $f$ be a distribution in $\mathcal{F}_{p}(\mathbb{R})$. Then $f$ is never equal to a sum of $r$-differences in $\mathcal{F}_{p}(\mathbb{R})$ if and only if for all $b_{1}, b_{2}, \ldots, b_{r} \in \mathbb{R}$ we have

$$
\int_{-\infty}^{\infty} \frac{|\widehat{f}(x)|^{2}}{\sum_{i=1}^{r} \mathrm{~d}_{\mathbb{Z}}\left(b_{i} x\right)^{p s}} d x=\infty .
$$

Proof. The proof is along the lines of Lemma 4.5. Details may be found in [7, Theorem 2] and [8, Proposition II.5.2].

Theorem 5.4 Let $1 \leq p<\infty$. Then there is a vector subspace $W$ of $\mathcal{F}_{p}(\mathbb{R})$ which has the following properties.

(i) $W \subseteq \bigcap_{s>0} \mathcal{D}_{s}\left(\mathcal{F}_{p}(\mathbb{R})\right)$.

(ii) If $s \geq 1 / p$, and if $\alpha: \mathbb{R} \longrightarrow \mathbb{C}$ is any $2 \pi$-periodic continuous function such that there are $c_{1}, c_{2}>0$ such that

$$
c_{1}|x|^{s} \leq|\alpha(x)| \leq c_{2}|x|^{s} \text { for all } x \in[-\pi, \pi],
$$

then any non-zero function $f$ in $W$ is a sum of $\operatorname{int}(p s)+1 \alpha$-differences, but $f$ is never the sum of $\operatorname{int}(p s) \alpha$-differences.

(iii) The algebraic dimension of $W$ is the cardinality of the continuum. 
Proof. The idea is to map the result of Theorem 4.8 across from $L^{2}(\mathbb{R})$ to $\mathcal{F}_{p}(\mathbb{R})$. To to this end, observe that if $f \in L^{2}(\mathbb{R})$ then $|\widehat{f}|^{2 / p} \in L^{p}(\mathbb{R})$. So, we may define a map $\Psi_{p}: L^{2}(\mathbb{R}) \longrightarrow \mathcal{F}_{p}(\mathbb{R})$ by

$$
\Psi_{p}(f)^{\curlywedge}=\mid \widehat{f}^{2 / p} .
$$

Now, let $\left\{f_{\Theta_{\gamma}}: \gamma \in \Gamma\right\}$ be the family of functions in $L^{2}(\mathbb{R})$ defined at the beginning of the proof of Theorem 4.8, and and define $g_{\gamma}=\Psi_{p}\left(f_{\Theta_{\gamma}}\right) \in \mathcal{F}_{p}(\mathbb{R})$ for each $\gamma \in \Gamma$. Let $q \in \mathbb{N}$ and let $\gamma_{1}, \gamma_{2}, \ldots, \gamma_{q}$ be distinct elements of $\Gamma$. Then, by (4.33), there is $A>0$ such that for all $i, j \in\{1,2, \ldots, q\}$ with $i \neq j$,

$$
\widehat{g}_{\gamma_{i}}(x) \widehat{g}_{\gamma_{j}}(x)=\left|\widehat{f}_{\Theta_{\gamma_{i}}}(x)\right|^{2 / p} \cdot\left|\widehat{f}_{\Theta_{\gamma_{j}}}(x)\right|^{2 / p}=0 \text {, for all } x \in[A, \infty) .
$$

Also, let $\beta_{1}, \beta_{2}, \ldots, \beta_{q} \in \mathbb{C}$ and let

$$
g=\sum_{k=1}^{q} \beta_{k} g_{\gamma_{k}} .
$$

Using (5.1), we now have that for all $r \in \mathbb{N}$ and for all $b_{1}, b_{2}, \ldots, b_{r} \in \mathbb{R}$,

$$
\begin{aligned}
& \int_{A}^{\infty} \frac{|\widehat{g}(x)|^{p}}{\sum_{i=1}^{r} \mathrm{~d}_{\mathbb{Z}}\left(b_{i} x\right)^{r}} d x=\int_{A}^{\infty} \frac{\left|\left(\sum_{k=1}^{q} \beta_{k} g_{\gamma_{k}}\right)^{-}(x)\right|^{p}}{\sum_{i=1}^{r} \mathrm{~d}_{\mathbb{Z}}\left(b_{i} x\right)^{r}} d x \\
& =\int_{A}^{\infty} \frac{\left|\sum_{k=1}^{q} \beta_{k} \widehat{g}_{\gamma_{k}}(x)\right|^{p}}{\sum_{i=1}^{r} \mathrm{~d}_{\mathbb{Z}}\left(b_{i} x\right)^{r}} d x \\
& =\int_{A}^{\infty} \frac{\sum_{k=1}^{q}\left|\beta_{k}\right|^{p}\left|\widehat{g}_{\gamma_{k}}(x)\right|^{p}}{\sum_{i=1}^{r} \mathrm{~d}_{\mathbb{Z}}\left(b_{i} x\right)^{r}} d x \\
& =\int_{A}^{\infty} \frac{\left.\sum_{k=1}^{q}\left|\beta_{k}\right|\right|^{p}\left|\widehat{f}_{\Theta_{\gamma_{k}}}(x)\right|^{2}}{\sum_{i=1}^{r} \mathrm{~d}_{\mathbb{Z}}\left(b_{i} x\right)^{r}} d x \\
& =\sum_{k=1}^{q}\left|\beta_{k}\right|^{p} \int_{A}^{\infty} \frac{\left|\widehat{f}_{\Theta_{\gamma_{k}}}(x)\right|^{2}}{\sum_{i=1}^{r} \mathrm{~d}_{\mathbb{Z}}\left(b_{i} x\right)^{r}} d x \\
& =\infty \text {, }
\end{aligned}
$$


by virtue of (4.13). It is immediate from (5.3) that the distributions in $\left\{g_{\gamma}: \gamma \in \Gamma\right\}$ are linearly independent in $\mathcal{F}_{p}(\mathbb{R})$. We take $W$ to be the vector subspace of $\mathcal{F}_{p}(\mathbb{R})$ which is spanned by the distributions $g_{\gamma}, \gamma \in \Gamma$. That is, the distributions in $W$ are those of the form $g$ given in (5.2). Thus, $W$ has the cardinality of the continuum, which establishes (iii). It remains to establish (i) and (ii).

We have by the construction of the functions $f_{\Theta}$ in Theorem 4.8 that for each $\gamma \in \Gamma$,

$$
\widehat{g}_{\gamma}(x)=\left|\widehat{f}_{\Theta_{\gamma}}(x)\right|^{2 / p}=0 \text {, for all } x \text { in some neighbourhood of } 0 .
$$

Consequently, by Theorem $5.1, g_{\Theta_{\gamma}}$ is in $\mathcal{D}_{s}\left(\mathcal{F}_{p}(\mathbb{R})\right)$ for all $\gamma \in \Gamma$ and it follows that $W$ is contained in $\cap_{s>0} \mathcal{D}_{s}\left(\mathcal{F}_{p}(\mathbb{R})\right)$. This proves (i).

Now, $s \geq 1 / p$ implies $\operatorname{int}(p s) \in \mathbb{N}$. So, in this case, take $g$ as in (5.2) and take $r=\operatorname{int}(p s)$ in (5.3). We then have

$$
\begin{aligned}
\int_{-\infty}^{\infty} \frac{|\widehat{g}(x)|^{p}}{\sum_{i=1}^{\operatorname{int}(p s)} \mathrm{d}_{\mathbb{Z}}\left(b_{i} x\right)^{p s}} d x & =\int_{-\infty}^{\infty} \frac{\left|\left(\sum_{k=1}^{q} \beta_{k} g_{\gamma_{k}}\right)^{\hat{(}(x)}\right|^{p}}{\sum_{i=1}^{\operatorname{int}(p s)} \mathrm{d}_{\mathbb{Z}}\left(b_{i} x\right)^{p s}} d x \\
& \geq \int_{-\infty}^{\infty} \frac{\left|\left(\sum_{k=1}^{q} \beta_{k} g_{\gamma_{k}}\right)^{-}(x)\right|^{p}}{\sum_{i=1}^{\operatorname{int}(p s)} \mathrm{d}_{\mathbb{Z}}\left(b_{i} x\right)^{\operatorname{int}(p s)}} d x \\
& \geq \int_{A}^{\infty} \frac{\left|\left(\sum_{k=1}^{q} \beta_{k} g_{\gamma_{k}}\right)^{-}(x)\right|^{p}}{\sum_{i=1}^{\operatorname{int}(p s)} \mathrm{d}_{\mathbb{Z}}\left(b_{i} x\right)^{\operatorname{int}(p s)}} d x \\
& =\infty .
\end{aligned}
$$

It follows from (5.4) and Lemma 5.3 that when $s \geq 1 / p$ no distribution $g$ in $W$ can be expressed as a sum of $\operatorname{int}(p s) \alpha$-differences in $\mathcal{F}_{p}(\mathbb{R})$. Also, as $g$ is in $W, g$ is a sum of $\operatorname{int}(p s)+1 \alpha$-differences by Theorem 5.2. This proves (ii).

\section{References}

[1] G. Barequet, A lower bound for Heilbronn's triangle problem in d dimensions, Proc. $10^{\text {th }}$ ACM-SIAM Symp. Discrete Algorithms, 1999, 76-81.

[2] J. Bourgain, Translation invariant forms on $L^{p}(G), 1<p<\infty$, Ann. Inst. Fourier (Grenoble), 36 (1986), 97-104.

[3] B. Johnson, A proof of the translation invariant form conjecture for $L^{2}(G)$, Bull. des Sciences. Math. 107 (1983), 301-310. 
[4] W. L. Lo, Difference Spaces on Locally Compact Groups, PhD thesis, University of Wollongong, 1996.

[5] G. H. Meisters, Some discontinuous translation invariant forms, J. Funct. Anal. 12 (1973), 199-210.

[6] G. H. Meisters and W. M. Schmidt, Translation invariant linear forms on $L^{2}(G)$ for compact abelian groups G, J. Funct. Anal. 11 (1972), 407-424.

[7] R. Nillsen, Banach spaces of functions and distributions characterized by singular integrals involving the Fourier transform, J. Funct. Anal. 110 (1992), 73-95.

[8] R. Nillsen, Difference Spaces and Invariant Linear Forms, Lecture Notes in Mathematics 1586, Heidelberg, New York: Springer-Verlag, 1994.

[9] R. Nillsen, Differentiate and make waves, Expos. Math. 14 (1996), 57-84.

[10] R. Nillsen and S. Okada, Function spaces and multiplier operators, Math. Annal. (to appear).

[11] W. Rudin, Fourier Analysis on Groups, 2nd ed., New York: McGraw-Hill, 1991.

[12] W. Schmidt, On a problem of Heilbronn, J. London Math. Soc. (2) 4 (1972), 545-550.

[13] T. Tao, From rotating needles to stability of waves: emerging connections between combinatorics, analysis and PDEs, Notices Amer. Math. Soc. 48 (2001), 294-303.

Email addresses

Rodney Nillsen:〈rodney_nillsen@uow.edu.au〉

Susumu Okada:〈okada@ics.mq.edu.au〉 